\title{
Simulation-Based Optimum Design of Sensor Arrays Using Multi- Objective Tabu Search
}

\author{
Zongyu Geng \\ West Virginia University
}

Follow this and additional works at: https://researchrepository.wvu.edu/etd

\section{Recommended Citation}

Geng, Zongyu, "Simulation-Based Optimum Design of Sensor Arrays Using Multi-Objective Tabu Search" (2010). Graduate Theses, Dissertations, and Problem Reports. 2173.

https://researchrepository.wvu.edu/etd/2173

This Thesis is protected by copyright and/or related rights. It has been brought to you by the The Research Repository @ WVU with permission from the rights-holder(s). You are free to use this Thesis in any way that is permitted by the copyright and related rights legislation that applies to your use. For other uses you must obtain permission from the rights-holder(s) directly, unless additional rights are indicated by a Creative Commons license in the record and/ or on the work itself. This Thesis has been accepted for inclusion in WVU Graduate Theses, Dissertations, and Problem Reports collection by an authorized administrator of The Research Repository @ WVU. For more information, please contact researchrepository@mail.wvu.edu. 


\title{
Simulation-Based Optimum Design of Sensor Arrays Using Multi-Objective Tabu Search
}

\section{Zongyu Geng}

Thesis submitted to the

\section{College of Engineering and Mineral Resources}

at West Virginia University

in partial fulfillment of the requirements

for the degree of

\author{
Master of Science \\ in \\ Industrial Engineering
}

\author{
Feng Yang, Ph.D., Chair \\ Nianqiang Wu, Ph.D., Co-Chair \\ Majid Jaraiedi, Ph.D. \\ Department of Industrial and Management Systems Engineering
}

\author{
Morgantown, West Virginia \\ 2010
}

Keywords: Sensor array, Design optimization, Multiple objective tabu search, Multivariate calibration, Sensor simulation, Selectivity and sensitivity

Copyright 2010 Zongyu Geng 


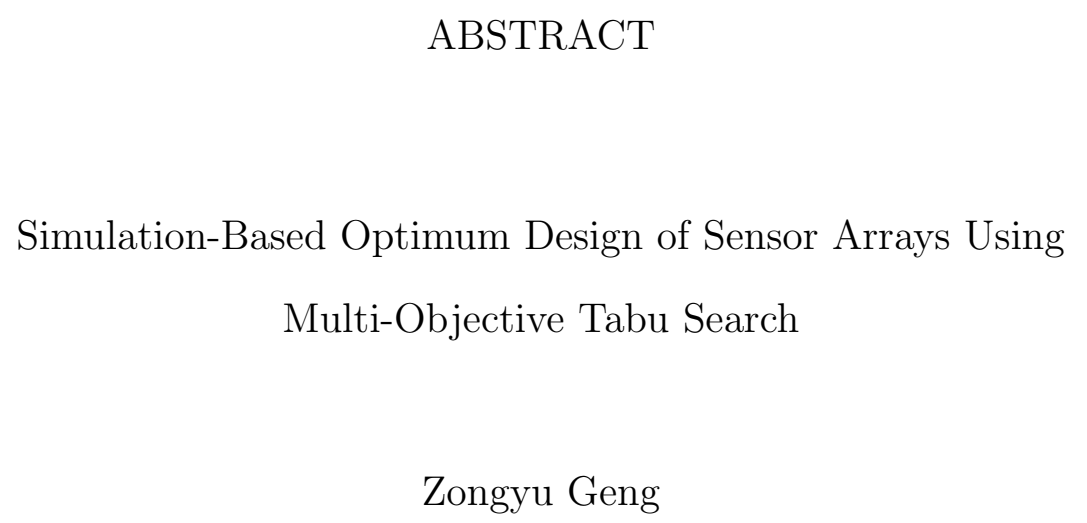

Zongyu Geng

This thesis is concerned with the optimum design of sensor arrays (i.e, electronic noses or tongues) using simulation experiments. The proposed design method adopts a set of new criteria, which more adequately represent the desirable properties of a sensor array in practice. A number of best non-dominated array designs are selected through a multiple objective (criteria) Tabu search algorithm. The evaluation of a candidate sensor array is based on its multivariate calibration model, which is efficiently estimated from well-designed simulation experiments. The method can be used to optimize the design of both linear and nonlinear sensor arrays. 


\section{ACKNOWLEDGMENTS}

I would like to thank my advisors, Dr. Feng Yang and Dr. Nianqiang Wu, for their great guidance, for giving me the opportunity to work on this project, which could not have been written without their encouragement and support. I am also thankful to Dr. Majid Jaraiedi for serving on my committee, and for his insightful ideas and assistance in preparing this thesis. 


\section{Contents}

List of Figures $\quad$ vi

List of Tables

List of Listings viii

1 Introduction $\quad 1$

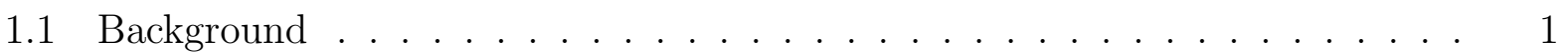

1.2 Problem Statement . . . . . . . . . . . . . . . . . . 2

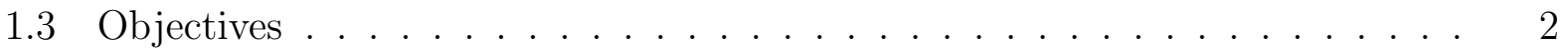

2 Literature Review $\quad 5$

2.1 Sensor Selection Criteria . . . . . . . . . . . . . . . . . 5

2.1.1 Evaluation of Sensor Arrays . . . . . . . . . . . . . . 5

2.1.2 Selectivity and Sensitivity . . . . . . . . . . . . 6

2.1.3 Error Criteria . . . . . . . . . . . . . . . 7

2.2 Multi-Objective Optimization Problems . . . . . . . . . . . . . . 8

2.2.1 Hansen's MOTS Method . . . . . . . . . . . . . . 8

2.2.2 Kulturel-Konak's MOTS Method . . . . . . . . . . . . . . 9

2.2.3 Baykasoglu's MOTS Method . . . . . . . . . . . . . 10

3 Methodology 11

3.1 Simulation-Based Multivariate Calibration . . . . . . . . . . . . . . . 12

3.1.1 Linear Multivariate Calibration . . . . . . . . . . . . . . . 12 
3.1.2 Nonlinear Multivariate Calibration . . . . . . . . . . . . . 12

3.2 Sensor Selection Criteria . . . . . . . . . . . . . . . . . . 14

3.2.1 Independence Across Individual Sensors . . . . . . . . . . . . . . . . 14

3.2.2 Sensitivity to Changes in Analytes . . . . . . . . . . . . . . . 16

3.2.3 Comparison with the MSE Criterion . . . . . . . . . . . . . 17

3.3 Array Optimization . . . . . . . . . . . . . . . 18

3.3.1 Sensor Array Optimization with Multi-objectives . . . . . . . . . 18

3.3.2 The Multi-Objective Tabu Search Algorithm . . . . . . . . . . . . . . 19

4 Empirical Results $\quad 23$

4.1 Simulation Model . . . . . . . . . . . . . . . . . . 23

4.1.1 The Linear Simulation Model . . . . . . . . . . . . . . . . . . . 24

4.1.2 The Nonlinear Simulation Model . . . . . . . . . . . . . . 26

4.2 Case 1: A Linear $\mathbf{c}-\mathbf{r}$ Relationship . . . . . . . . . . . . . . . . 27

4.3 Case 2: A Nonlinear $\mathbf{c}-\mathbf{r}$ Relationship . . . . . . . . . . . . . . . . 30

4.4 Efficiency of the modified MOTS algorithm . . . . . . . . . . . . . 33

5 Conclusion $\quad 36$

$\begin{array}{ll}\text { References } & 37\end{array}$

A The Matlab Program for the Simulation Model of Chemiresistors 40

A.1 The Linear Simulation Model for Chemiresistors . . . . . . . . . . . . . . 40

A.2 The Nonlinear Simulation Model for Chemiresistors . . . . . . . . . . . . . 42

B The Database of 10 polymers and 9 analytes 44

C The Matlab Program Applying the Modified MOTS Algorithm 46 


\section{List of Figures}

3.1 The flowchart of the modified MOTS algorithm. . . . . . . . . . . . 21

4.1 The relationship between the two sub-models for the chemiresistor. . . . . . 24

4.2 Activity of toluene with different vapor concentrations of methanol. . . . . . 27

4.3 Central composite design (CCD) for nonlinear calibration model. . . . . . . . 31

4.4 The distribution of the number of Pareto optimums found (Modified MOTS). 34

4.5 The distribution of the number of configurations evaluated (Modified MOTS). 35 


\section{List of Tables}

4.1 Candidate polymers for the sensor array. . . . . . . . . . . . . . 28

4.2 The true Pareto optimal set from an exhaustive search . . . . . . . . . . . 29

4.3 The performance of the modified MOTS algorithm. . . . . . . . . . . . 30

4.4 The Pareto optimal set (nonlinear case). . . . . . . . . . . . . . . . . . 33

4.5 The improvement of search results after our modification. . . . . . . . . 33

B.1 The Database of 10 polymers and 9 analytes. . . . . . . . . . . . 45 


\section{List of Listings}

A.1 The Matlab program for the linear simulation model of chemiresistors . . . . 40

A.2 The Flory-Huggins equation to be solved in the nonlinear simulation model . 42

A.3 The Matlab program for the nonlinear simulation model of chemiresistors . . 43

C.1 Criteria used in array optimization . . . . . . . . . . . . . 46

C.2 The Matlab program applying the modified MOTS algorithm . . . . . . . . . 48 


\section{Chapter 1}

\section{Introduction}

\section{$1.1 \quad$ Background}

A sensor is a device that measures a physical quantity and converts it into a signal which can be read by an observer or by an instrument. For example, a mercury-in-glass thermometer converts the measured temperature into expansion and contraction of a liquid which can be read on a calibrated glass tube. There are innumerable applications for sensors in daily lives, industry and academic research. This work focuses on chemical sensors which are sensitive to changes in their environment, and give quantitative or qualitative feedback of these changes.

Most chemical sensors are small, probe-like devices. They can be used to monitor dynamic fluctuations of sample species in real time or provide feedback for control purposes. However, in real applications, the responsive chemicals employed in chemical sensors can respond to various analytes in their surroundings. Thus, the problem of cross selectivity bothers scientists and engineers: the response of each individual sensor reveals mixed information of multiple analytes, other than the exact information of a specific analyte.

To solve the problem of cross selectivity, the conventional approach is based on the utility of new materials synthesized to build a specific receptor which responds to the analyte strongly and selectively. The disadvantage is that this kind of sensor can only be useful to detect a specific analyte but not proper for identifying complex mixtures. Besides, the synthesization of new materials is complex and time-consuming.

Sensor arrays, also known as electronic noses, is another approach, with each sensor responds to various analytes, simulating the mammalian olfactory system. Gathering in- 
formation from different sensors, allows a sensor array to respond and distinguish a large number of analytes. No sensor is required to respond highly selectively to a specific analyte, and after the sensor array is calibrated, it can provide both quantitative and qualitative information of the composition of a mixture.

Nowadays, sensor arrays are finding wide applications in areas such as emission monitoring, quality control, home safety alarms, environmental monitoring, medical diagnosis, and more [1]. More improvements are needed for both economic and social benefits, which become the motivation of this work.

\subsection{Problem Statement}

One of the major advantages of sensor arrays is their ability to adapt to specific measurement problems. By carefully selecting component sensors, a sensor array is able to identify/quan-

tify a number of target analytes for a particular application. However, when approaching a new sensing problem, usually a large variety of sensor elements are available for selection to form the desired array. How to design a sensor array that has the lowest complexity and best performance is the subject of "array optimization", the primary interest of this thesis.

The main challenge comes from the existence of a vast number of possible configurations (combinations), which makes it impossible to test each single configuration by real experiments. The unbearable cost/time likely involved in optimizing sensor arrays via real experiments can be substantially mitigated-if not completely overcome-by the use of simulation, a computer model that mimics sensor responses in various analyte environments.

\subsection{Objectives}

This thesis intends to take advantage of the benefit of simulation, and to adapt statistical methods and optimization heuristics for the optimum designs of sensor arrays.

There are two main objectives of this research: 
1. To find new criteria for the evaluation of sensor arrays Although, many criteria have been discussed in current literature, we still aim to find some new criteria which more adequately reflect the desirable properties of a sensor array. In addition, these new criteria will also provide the potential to substantially reduce the candidate pool of array configurations by utilizing prior knowledge.

2. To optimize the selection of sensor arrays The selection of sensor configurations is very critical in the design of an effective sensor array. Based on the criteria we introduce, a multi-objective Tabu search algorithm will be adapted to search for a set of best configurations that are diverse and non-dominated to each other.

Besides, our method will also be distinct from the existing array optimization work in the following aspects: (i) Design of experiments (DOE) techniques will be used to carry out simulation experiments efficiently for the calibration and evaluation of the candidate arrays. (ii) The proposed method can be applied to optimize both linear and nonlinear sensor arrays.

The proposed work utilizes computer simulation, and aims to efficiently select among a potentially large number of sensor combinations the most promising array configurations for a practical application. The selected small set of array designs can be further evaluated through other venues such as real experiments, which is beyond the scope of the current work. This is similar in spirit to Lei's research [2], which shares the same objective but differs in the two aspects above. To illustrate the proposed method of array optimization, the class of sensors investigated by Lei et al. [2], conductive polymer composite sensors, are used as examples in this work.

The remainder of the thesis is organized as follows. Chapter 2 reviews current research papers on topics of concern in this work. Chapter 3 details the simulation-based method for optimizing a sensor array. In Chapter 4, the proposed method is applied to the design of arrays consisting of conductive polymer composite sensors. A conclusion is given in 
Chapter 5. Besides, all the calculation programs and the database we used could be found in the Appendices. 


\section{Chapter 2}

\section{Literature Review}

\subsection{Sensor Selection Criteria}

To build a sensor array, there must be a lot of alternatives. Therefore, the question is: "What kind of configurations would be the optimal selection?" Certain criteria are needed to evaluate different configurations during the selection of optimal sensor arrays.

\subsubsection{Evaluation of Sensor Arrays}

For a better description of the sensor array selection algorithm, some notations have to be

defined. Given a sensor array having $p$ different sensors, the response output to a mixture of $m$ analytes is shown as

$$
\mathbf{r}=\mathbf{S c}+\mathbf{e}
$$

where $\mathbf{r}$ is a $p \times 1$ vector of responses, $\mathbf{c}$ is a $m \times 1$ vector of concentrations of the $m$ analytes (in a mixture), $\mathbf{e}$ is the $p \times 1$ vector of errors, and $\mathbf{S}$ is the $p \times m$ sensitivity matrix of the sensor array to the analytes. Assuming $p \geq m$, each element $s_{i j}$ in the sensitivity matrix is the response of the $i^{\text {th }}$ sensor in the array to the $j^{\text {th }}$ analyte in the sample. The values in the column of $\mathbf{S}$ are responses of the sensor elements to the pure components at unit concentration. Given a response $\mathbf{r}$ to a sample of unknown concentration but known components, a prediction for $\mathbf{c}$ can be obtained by

$$
\mathbf{c}=\mathbf{S}^{+}(\mathbf{r}-\mathbf{e})
$$


where $\mathbf{S}^{+}\left(=\left(\mathbf{S}^{T} \mathbf{S}\right)^{-1} \mathbf{S}^{T}\right)$ is the pseudoinverse of matrix. If there are no errors $\mathbf{e}$ in both the calibration and the measurement, the sensitivity matrix $\mathbf{S}$ would determine the performance of sensor arrays.

\subsubsection{Selectivity and Sensitivity}

There are two features of merit which have been widely used to measure the performance of sensor arrays:

1. Sensitivity: how strongly the sensors respond to the target analytes.

2. Selectivity: how well a sensor array can distinguish one analyte from another.

The most popular definition of the selectivity measures was given by Lorber [3]. In the evaluation of sensor arrays, the orthogonal part, $\mathbf{s}_{k}^{*}$, of the response vector $\mathbf{s}_{k}$ (which corresponds to the $k^{\text {th }}$ analyte in the sensitivity matrix) is

$$
\mathbf{s}_{k}^{*}=\left(\mathbf{I}-\mathbf{S}_{k} \mathbf{S}_{k}^{+}\right) \mathbf{s}_{k}
$$

where $\mathbf{s}_{k}$ is the $p \times(m-1)$ matrix of analytes except the $k^{t h}$ component. This $\mathbf{s}_{k}^{*}$ is called the net analyte signal. The sensor array whose $\mathbf{S}$-matrix produces larger values in $\mathbf{s}_{k}$, could perform better in measuring the concentration of analytes. Lorber quantifies the selectivity for analyte $k$ as the ration of the norm of the net analyte signal to the norm of the total data for component $k$ as

$$
\xi_{k}=\left\|\mathbf{s}_{k}^{*}\right\| /\left\|\mathbf{s}_{k}\right\|
$$

If the $\mathbf{S}$-matrix were a diagonal matrix, each of the $\xi_{k}$ values would be unity; but if the sensor arrays were not selective for analyte $k$, then $\xi_{k}$ would be near zero.

A measurement of the sensitivity of analyte $j$ can be calculated as shown below [4].

$$
\mathrm{RSS}_{j}=\sqrt{\sum_{i=1, \ldots, p} S_{i j}^{2}}
$$


In some literature e.g. [4], the sum of $\operatorname{RSS}_{j}\left(=\sum_{i=1, \ldots, m} \mathrm{RSS}_{j}\right)$ is used to measure the overall sensitivity of a sensor array. However, this method can not avoid the array designs where sensor arrays are highly sensitive to some analytes of interest whereas being insensitive to the others.

\subsubsection{Error Criteria}

The fundamental criterion by which a model for quantitative analysis should be evaluated is the magnitude of the difference between the predicted values and true values. The mean square error (MSE) is a popular criterion.

$$
\mathrm{MSE}=\mathrm{E}(\mathbf{c}-\widehat{\mathbf{c}})^{2}
$$

Here, $\mathbf{c}$ denotes the true concentration of sample analytes, and $\widehat{\mathbf{c}}$ represents the predicted concentration obtained from the sensor array device plus the corresponding calibration model. MSE incorporates various types of errors: the bias of the calibration model and the random noise in the calibration and prediction data [5]. It has been shown that when the sensitivity and selectivity are good, the deviation of the predicted concentration from the true concentration is small, which means that the total error is small [6]. Therefore, the minimum MSE value implies the maximum of the combination of selectivity and sensitivity.

In Lei's work [2], the best sensor array is constructed by selecting the set of sensors which has the minimum MSE value. The MSE value is calculated based on the information of the sensitivity matrix S. In Section 3.2, we will compare this MSE criterion with the two criteria we use in our array design.

Most current literature in sensor selection focus on finding the most informative criterion, which then comes with a single-objective optimization problem. To the best of our knowledge, few works have been done to optimize sensor designs with multi-criteria. 


\subsection{Multi-Objective Optimization Problems}

As the name suggests, a multi-objective optimization problem (MOOP) deals with more than one objective function. Generally, there is no any single solution, which can simultaneously provide the optimal value on all objectives. In single-objective optimization, only one optimal solution needs to be found. However, there are two goals in multi-objective optimization [7]:

1. To find a set of solutions as close as possible to the Pareto-optimal front.

2. To find a set of solutions as diverse as possible.

The Pareto-optimal front is also known as the non-dominated set of solutions, where no objective function can be improved without worsening at least one other objective function. In short, the first task in an ideal multi-objective optimization is to find all possible solutions along the pareto-optimal front.

In some MOOP survey papers, the application of metaheuristics, such as simulated annealing, tabu search and genetic algorithms, are recommended, since they are relatively easy to implement and generate good solutions in comparatively less time than classical optimization methods. Although Tabu Search is mentioned in these survey papers, it has rarely been the primary focus of multi-objective optimization approaches. A recent overview of metaheuristics methods for MOOP [8] points out that only about $6 \%$ of studies utilized Tabu Search while 70\%, the majority, employed GA.

During a brief survey of current multi-objective Tabu Search approach (MOTS), we find out that there are three typical and different kinds of methods, developed by Hansen [9], Kulturel-Konak [10], and Baykasoglu [11] respectively.

\subsubsection{Hansen's MOTS Method}

The Hansen's method [9] works with a set of solutions searching for Pareto optimal solutions in parallel. To find the best candidate in the neighborhood of each current solution, the fitness was the weighted average of the objectives. Since the weights were important, they 
were dynamically updated so that unexplored regions of the Pareto front become attractive. The results of applications indicated that the procedure obtained better solutions than Pareto Simulated Annealing(PSA).

These types of methods depend on combining the set of objectives into a single objective using a weighting scheme. Although the approach can be effective, it has several inherent difficulties:

1. it is often not known how much importance should be given to each objective (i.e., weighting) in advance of conducting (or during) the optimization procedure.

2. The weighted-sum approach cannot identify all points in a trade-off surface of nonconvex and discontinuous solutions spaces.

3. a problem of scaling among objectives can occur.

\subsubsection{Kulturel-Konak's MOTS Method}

This selection method [10] eliminates two major problems of simple multi-objective methods, a priori weighting and scaling of objective.

The idea here is simple, but very general and easy to implement. For each iteration, a single objective is selected to become active from the set of all objectives using a multinomial probability mass function. In the simplest case, the probabilities are equal. Generally, when higher probability is given to certain objective, more solutions with higher performance in this objective could be found. But the range of excellent solutions stays wide even with dramatic changes in probabilities.

Kulturel-Konak demonstrated that his methods can be applied to up to three objectives, and it is quite effective at identifying a wide Pareto front, compared to Hansen's MOTS Method [9]. The empirical results show that the simplest version (i.e. equal probabilities for all objectives) worked the best. 


\subsubsection{Baykasoglu's MOTS Method}

Baykasoglu's main contribution to the Multi-objective Tabu Search method comes with the utilization of two additional lists: the Pareto list and the candidate list [11].

1. The Pareto list: collects selected non-dominated solutions found by the algorithm.

2. The candidate list: collects all other non-dominated solutions, which are not selected as Pareto optimal solutions in the current iteration. These solutions may become seed solutions if they maintain their non-dominated status in later iterations. The candidate list also works as a source of diversion, which is very important to avoid local optima.

Thus, there is also no need to apply a weighting scheme in Baykasoglu's Method. The objective functions are not combined into a single function by using some kind of utility functions. Moreover, there is not any extra Tabu Search parameter to set for the running the optimization procedure. In contrast to original Tabu Search algorithm, the iterative update of the two additional lists (the Pareto list and the candidate list) provides a well-structured and aggressive route to search promising regions.

Baykasoglu demonstrated that his method could be applied to MOOP with continuous variables, up to three objectives, and the quality of solutions obtained from his method is acceptable compared to GAs. The performance can also be improved by using more advanced neighborhood functions and adaptive strategies.

Disadvantages exist, such as (i) the fixed number of neighbors searched each iteration (ii) easy to be halted (iii) the diversion effect of the candidate list is limited. The details will be discussed with empirical results in Section 4.4. 


\section{Chapter 3}

\section{Methodology}

Suppose that the sensor array is designed to quantify $p$ different analytes, the concentrations of which are represented by the $p \times 1$ vector $\mathbf{c}^{\prime}=\left(c_{1}, c_{2}, \ldots, c_{p}\right)$. Let $q$ be the number of sensors included in the array, and denote the sensor responses by the $q \times 1$ vector $\mathbf{r}^{\prime}=\left(r_{1}, r_{2}, \ldots, r_{q}\right)$. Generally, it is required that $q \geq p$, since otherwise $\mathbf{c}$ cannot be identified even from an error free $\mathbf{r}[12]$. In this work, we assume $q=p$, i.e., the number of sensors is equal to its minimum, the number of different analytes to be detected. This assumption is made out of the following considerations. First, the purpose of design here is to find array configurations that are adequate to quantify the analytes of interest as well as of the lowest complexity, which in this context implies the smallest number of sensors. Second, in cases where more than the minimum number of sensors are needed, techniques such as principal component analysis can be used to cut the dimension of the sensor data down to the minimum $p$.

Assuming $p=q$, the relationship between $\mathbf{c}$ and $\mathbf{r}$ can be approximated by the multivariate calibration model:

$$
r_{i}=f_{i}\left(\mathbf{c}, \boldsymbol{\theta}_{i}\right), \quad i=1,2, \ldots, p
$$

where $\boldsymbol{\theta}_{i}$ represents the unknown parameter vector. The objective of array optimization is to identify $p$ sensors, associated with which a good calibration model $\left\{f_{1}, f_{2}, \ldots, f_{p}\right\}$ can be

obtained and used for accurate and precise prediction of the analyte concentrations of an unknown sample. 
Note that in this thesis, two different models were developed to quantify the $\mathbf{c}-\mathbf{r}$ relationship. One is the simulation model, a sequence of complicated mathematical equations (Section 4.1). The other is a calibration model denoted as (3.1), which is linear/nonlinear derivable equations of straightforward functional forms. In this work, to optimize array designs without actually building the sensors, simulation models were developed quantifying the $\mathbf{c}-\mathbf{r}$ relationship for sensor arrays with sufficient accuracy. From simulation data, calibration models were obtained providing a good and simple functional approximation to the underlying relationship implied by simulation. The evaluation of each array configuration is based on its multivariate calibration models, as will be seen in Section 3.2.

\subsection{Simulation-Based Multivariate Calibration}

As already explained, the calibration model for a sensor array can be estimated from the data collected by running the simulation. We discuss two cases, linear and nonlinear sensor arrays.

\subsubsection{Linear Multivariate Calibration}

If the sensor responses are linearly dependent upon the concentration of analytes, then a calibration model of the form $\mathbf{r}=\mathbf{A c}$ is adequate to describe the $\mathbf{c}-\mathbf{r}$ relationship, with $\mathbf{A}=\left[A_{i j}\right]_{p \times p}$ being a square matrix estimated from simulation data. Each element $A_{i j}$ is the

response of the $i^{\text {th }}$ sensor in the array to the $j^{\text {th }}$ analyte in the sample. The $j^{\text {th }}$ column of the matrix, $A_{\cdot j}$, includes the responses of the $p$ sensors to the $j^{\text {th }}$ pure component analyte.

\subsubsection{Nonlinear Multivariate Calibration}

The sensor arrays would be much more difficult to calibrate if the dependence of sensor responses upon the multiple analyte concentrations is nonlinear. The nonlinearity mainly stems from two sources: (i) the presence of one analyte affects the way a sensor component 
reacts to another analyte; (ii) over certain concentration range (typically at relatively high concentration levels), the sensor responds to pure analyte following a nonlinear pattern.

Nonlinear calibration, as general nonlinear regression, is difficult due to the various complications involved in model identification, convergence of nonlinear fitting, etc. [13]. In the literature, a variety of models such as artificial neural nets (e.g., [14]) have been used to calibrate sensor arrays. In such works, efforts are rarely made to provide guidance on the design of experiments (i.e., the efficient data collection), or to ensure that a good model of the simplest functional form is achieved. This is mainly because these modeling issues are difficult to address for complicated nonlinear models. In this thesis, our simulation-based multivariate calibration is to assist the design of sensor arrays at relatively early stage, rather than to provide a highly accurate $\mathbf{c}-\mathbf{r}$ model which can well predict sample components from sensor responses in operational use. In light of this, we chose to use a full quadratic model (3.2), instead of complicated nonlinear models, to approximate the $\mathbf{c}-\mathbf{r}$ relationship.

$$
\begin{aligned}
r_{i} & =\sum_{m=1}^{p} \theta_{m}^{(i)} c_{m}+\sum_{m=1}^{p} \theta_{m, m}^{(i)} c_{m}^{2}+\sum_{m=1}^{p-1} \sum_{n=m+1}^{p} \theta_{m, n}^{(i)} c_{m} c_{n} \\
i & =1,2, \ldots, p
\end{aligned}
$$

Here, $\boldsymbol{\theta}^{(i)}=\left(\theta_{1}^{(i)}, \ldots, \theta_{p}^{(i)} ; \theta_{1,1}^{(i)}, \ldots, \theta_{p, p}^{(i)} ; \theta_{1,2}^{(i)}, \theta_{1,3}^{(i)}, \ldots, \theta_{p-1, p}^{(i)}\right)$ represents the unknown parameters to be estimated. The interaction terms $c_{m} c_{n}(m=1, \ldots, p-1 ; n=m+1, \ldots, p)$ intend to capture the possible effects on sensor responses due to the coexistence of two analytes. The quadratic terms are meant to capture the curvature effects of changing an analyte concentration upon sensor responses. Thus, model (3.2) is able to describe the main characteristics of the $\mathbf{c}-\mathbf{r}$ surfaces. At the same time, (3.2) is linear with respect to the unknown parameters and thus is much easier to estimate than its complicated counterparts.

To calibrate a sensor array using a model of the form (3.2), simulation experiments will be carried out at certain values of $\mathbf{c}$ following the central composite design (CCD), which was particularly developed to efficiently collect data for the estimation of a full quadratic 
model [15]. A graphical illustration of the CCD for a two-analyte case is given in Figure 4.3. Once the simulation data has been collected, multivariate least square regression [16] will be performed to obtain the fitted calibration model (3.2).

\subsection{Sensor Selection Criteria}

In this section, we introduce the criteria used to evaluate the array configurations based on their multivariate calibration models.

\subsubsection{Independence Across Individual Sensors}

An ideal sensor array consists of $q=p$ sensors with each component sensor reacting to changes in one of the $p$ analytes exclusively. For such an ideal array, the calibration model (3.1) can be written as follows (assuming linearity for the model):

$$
\left(\begin{array}{c}
r_{1} \\
r_{2} \\
\vdots \\
r_{p}
\end{array}\right)=\left(\begin{array}{cccc}
a_{11} & 0 & \cdots & 0 \\
0 & a_{22} & \cdots & 0 \\
\vdots & \ddots & \ddots & 0 \\
0 & 0 & \cdots & a_{p p}
\end{array}\right)\left(\begin{array}{c}
c_{1} \\
c_{2} \\
\vdots \\
c_{p}
\end{array}\right)
$$

In the ideal case represented by (3.3), each individual sensor reacts exclusively to its corresponding analyte independent of the existence of other analytes. Whereas practically, almost all sensors react to multiple analytes to some extent, in which case we need to know the degree of independence among individual sensors in order to evaluate the array configuration. Here, we use the measures of independence developed by Suh[17].

In [17], two measures, reangularity $R$ and semangularity $S$, were used conjointly to evaluate the functional independence of design alternatives, and they are applicable to both linear and nonlinear design problems. In the general setting of (3.1), the measures $R$ and $S$ 
are computed based on the first derivative matrix:

$$
\mathbf{D}=D_{i j}=\left[\frac{\partial r_{i}}{\partial c_{j}}\right]_{p \times p} \quad i=1, \ldots, p ; j=1, \ldots, p
$$

The two measures are defined as follows:

$$
R=\prod_{\substack{m=1, \ldots, p-1 ; \\ n=m+1, \ldots, p}}\left(1-\frac{\left(\sum_{k=1, \ldots, p} D_{k, m} D_{k, n}\right)^{2}}{\left(\sum_{k=1, \ldots, p} D_{k, m}^{2}\right)\left(\sum_{k=1, \ldots, p} D_{k, n}^{2}\right)}\right)^{1 / 2}
$$

and

$$
S=\prod_{m=1, \ldots, p} \frac{\left|D_{m, m}\right|}{\left(\sum_{n=1, \ldots, p} D_{m, n}^{2}\right)^{1 / 2}}
$$

Reangularity (3.5) measures the degree of orthogonality of the columns in the $\mathbf{D}$ matrix. The summation in the denominator of (3.5) are normalization factors, the squared magnitudes of columns; the summation in the numerator is the dot product of the $i^{\text {th }}$ and $j^{\text {th }}$ columns. Thus, the fraction term can be considered as the cosine of the angle between the $i^{\text {th }}$ and $j^{\text {th }}$ columns; and the reangularity is the absolute value of the product of the sines of the angles between all pairs of columns in the $\mathbf{D}$ matrix. The measure $R$ achieves its maximum and most desirable value, unity, when the columns are mutually orthogonal. The reangularity measures the ability of a sensor array to discriminate between all the pairs of analytes, and thus is similar in spirit to the selectivity measures used in the chemometrics literature, among which the definition given by Lorber [3] may be the most popular one.

Semangularity (3.6) measures the degree to which each sensor reacts to one and only one analyte. When all off-diagonal elements are zero, the $S$ reaches its maximum and most desirable value, unity. The design quality embodied by the metric $S$ has been largely ignored in the literature of array optimization, regardless of the clear benefits of having $S$ close to one. First, from the perspective of statistical modeling, a calibration model that associates with a diagonal (or close to diagonal) matrix $\mathbf{D}$, as defined in (3.4), involves less number of unknown model parameters to be estimated since $\mathbf{D}$ includes less non-zeros elements. This 
implies that the estimation of such a calibration model would require a smaller sample, and hence save substantial time/cost for data collection. Second, by designing an array in such a way that each sensor is particularly responsive to one analyte, it makes it easy to adapt the current array design to new applications aiming to detect different analyte combinations.

As already mentioned, $R$ and $S$ are joint measures of the degree of independence among the individual sensors included in an array. For an ideal sensor array, both $R$ and $S$ equal to 1, their maximum. When the design deviates from the ideal, the closer the values of $R$ and $S$ and the closer each of them is to 1 , the more functional independent is the sensor system [17]. To simultaneously minimize the closeness of $R$ and $S$ as well as maximize their individual values, we adopt the integrated criterion proposed by [18],

$$
I=\frac{R+S}{R \cdot S}
$$

which is to be minimized. The criterion $I$ and the sensitivity measure introduced next in Section 3.2.2 will be used as the two criteria to be optimized in our array selection.

\subsubsection{Sensitivity to Changes in Analytes}

Sensitivity measures how strongly the sensors respond to analytes of interest. Let $D_{i j}$ be as defined in (3.4), then the sensitivity of a sensor array to changes in analyte $j$ can be measured as the following root sum square (RSS):

$$
\mathrm{RSS}_{j}=\sqrt{\sum_{i=1, \ldots, p} D_{i j}^{2}}
$$

We define the overall array sensitivity to all the $p$ analytes of interest as

$$
\operatorname{MRSS}=\min _{j=1, \ldots, p} \operatorname{RSS}_{j}
$$


The sensitivity measure (3.9) is defined as the minimum of the array sensitivity to individual analytes, not the sum of $\mathrm{RSS}_{j}$ used in most literatures. This is to avoid array designs that are highly sensitive to some analytes of interest whereas being insensitive to the others.

\subsubsection{Comparison with the MSE Criterion}

In the evaluation of sensor arrays, we use the two criteria defined above, the independence measure $I$, which integrates $R$ and $S$, and the sensitivity measure MRSS. The purpose of developing a sensor array is for it to be able to quantify analyte mixtures when integrated with its calibration model. So the ultimate criterion for assessing a sensor array should be the ability to accurately predict the analyte concentrations of unknown samples, the measure of which is the well recognized mean square error (MSE):

$$
\mathrm{MSE}=\mathrm{E}(\mathbf{c}-\widehat{\mathbf{c}})^{2}
$$

Here, $\mathbf{c}$ denotes the true concentration of sample analytes, and $\widehat{\mathbf{c}}$ represents the predicted concentration obtained from the sensor array device plus the corresponding calibration model. MSE incorporates various types of errors: the bias of the calibration model and the random noise in the calibration and prediction data [5]. Although reflecting the fundamental requirement of sensor arrays, MSE is difficult to evaluate in practice. To estimate (3.10), the difference $\mathbf{c}-\widehat{\mathbf{c}}$ needs to be evaluated via real experiments in a wide range of (if not all possible) values of $\mathbf{c}[5]$, which is unrealistically expensive in terms of time and cost.

Due to the difficulties involved with MSE, alternative criteria have been developed. It has been shown that when the sensitivity (measured by MRSS) and selectivity (measured by $R$ ) are good, the deviation of the predicted concentration from the true concentration is small [6]. In our design evaluation, we include an additional measure $S$, the benefits of which have been explained in Section 3.2.1. 


\subsection{Array Optimization}

Different individual sensors can be selected to construct the sensor array which will be used to quantify multiple target analytes. Thus, scientists and engineers must answer the questions: How does one choose the combination of sensors in the sensor array? Some sensor sets must be better than others, so which set is the best? In this part, we describe the sensor array optimization problem in Section 3.3.1, and discuss our modified MOTS method used to solve this optimization problem in Section 3.3.2.

\subsubsection{Sensor Array Optimization with Multi-objectives}

Suppose that $p$ sensors are to be chosen out of a total of $K$ possible ones to quantify $p$ substances. By the criteria introduced in Section 3.2, our array optimization problem can be stated as follows:

Objective 1: $\min _{\mathbf{x}} I(\mathbf{x})$

Objective 2: $\max _{\mathbf{x}} \operatorname{MRSS}(\mathbf{x})$

$$
\begin{gathered}
\text { Subject to } x_{i}=0 \text { or } 1, \quad i=1,2, \ldots, K \\
\sum_{i=1}^{K} x_{i}=p .
\end{gathered}
$$

This is a multi-objective optimization (MOO) problem, where the decision variables are denoted as the vector $\mathbf{x}$. We have:

$$
\left\{\begin{array}{l}
x_{i}=1, \text { if the } i^{t h} \text { sensor is selected for the array; } \\
x_{i}=0 \text {, otherwise. }
\end{array}\right.
$$

The $I$ and MRSS metrics for a certain sensor array depend on the decision vector $\mathbf{x}$, and can be evaluated based on its multivariate calibration models estimated from simulation experiments (Sections 3.1 and 3.2).

Generally, there does not exist any single solution which can simultaneously provide the optimal value on all the objectives, and thus it is of interest to generate a set of non- 
dominated solutions, where no objective can be improved without worsening at least one other objective. The set of all non-dominated solutions is referred to as the Pareto optimal front [7]. Our goal of array optimization is to find a set of solutions to (3.11) as close as possible to the Pareto optimal front.

\subsubsection{The Multi-Objective Tabu Search Algorithm}

To solve (3.11) by an exhaustive search, a total of $\left(\begin{array}{c}K \\ p\end{array}\right)$ different $p$-sensor array configurations have to be evaluated. The number of all the possible configurations is typically large, and it could be extremely time-consuming, if not impossible, to explore them all even with computer simulation. In light of this, the methods of metaheuristics, such as simulated annealing(SA), tabu search and genetic algorithms(GA), have been widely applied in multi-objective optimization problems, since they are relatively easy to implement and generate good solutions in comparatively less time than classical optimization methods. Several heuristics selection methods have been implemented and compared to optimize wavelength/array selection [19]. Tabu Search was shown to be a valuable alternative to SA and GAs. The Tabu Search method is preferred to other heuristics methods in our application by the following reasons. (i) The definition of a neighborhood of a solution is very clear. (ii) In contrast to SA and GAs, Tabu Search is an iterative deterministic global optimization method, and relatively new in analytical chemistry. It examines the search space in a well-structured way using memory to keep track of regions already visited.

Thus, in this work, we adapted an optimization heuristics, the multi-objective tabu search (MOTS) algorithm proposed by Baykasoglu [11], to search for the Pareto optimal front of (3.11).

Our MOTS algorithm, outlined in Figure 3.1, is a modified version of the one developed by Baykasoglu [11]. Figure 3.1 is adapted from the "the flowchart of MOTS algorithm" in [11]. Comparing to other MOTS (e.g., [9], [10]), Baykasoglu's algorithm does not involve 
a weighting scheme for different objectives, and requires no extra parameters to be specified other than those in the original single-objective tabu search algorithm [7] [9].

It is fairly straightforward to apply Baykasoglu's MOTS algorithm on the optimum design of sensor arrays, where the search space is clearly defined and a neighborhood solution can be easily generated by replacing one sensor with another. However, in our experience, as shown in Section 4.2, running Baykasoglu's algorithm directly often leads to premature termination of the search with two or less Pareto optimums in the solution set. In light of this, we incorporate in Baykasoglu's algorithm a modification, which is given in the dotted square in Figure 3.1. The modified MOTS leads to substantial improvement in the sense that more Pareto optimums can be found. (The detailed comparisons are given in Section 4.2).

As can be seen from Figure 3.1, two actions are taken to avoid the premature termination of the algorithm: (i) temporarily increase the size of the neighborhood, and (ii) empty the tabu list. Both of them provide a more aggressive search scheme when the current neighborhood is not that promising. Since this scheme requires more candidate evaluations, we may want to control the times of using it depending on the total computational time available at hand.

The resulting solution set from our modified MOTS is close to the Pareto optimal front, and provides a number of array designs that are non-dominated to each other and superior in terms of both criteria $I$ and MRSS. These selected arrays can then be assessed by weighing the relative importance of the different objectives in (3.11); also, they can be further evaluated based on other qualities such as cost and robustness in various environments, and/or examined through real experiments. To our knowledge, most of the array optimization work in the literature treated the optimum design problem as a single-objective one, and selected best alternatives based on a single criterion or a single weighted average of multiple criteria (e.g., [20] [21]). Comparing to the single-objective optimization problems, our MOO leads to a better design pool containing diverse configurations that perform best in terms of multiple criteria simultaneously-in the sense of Pareto optimality. 


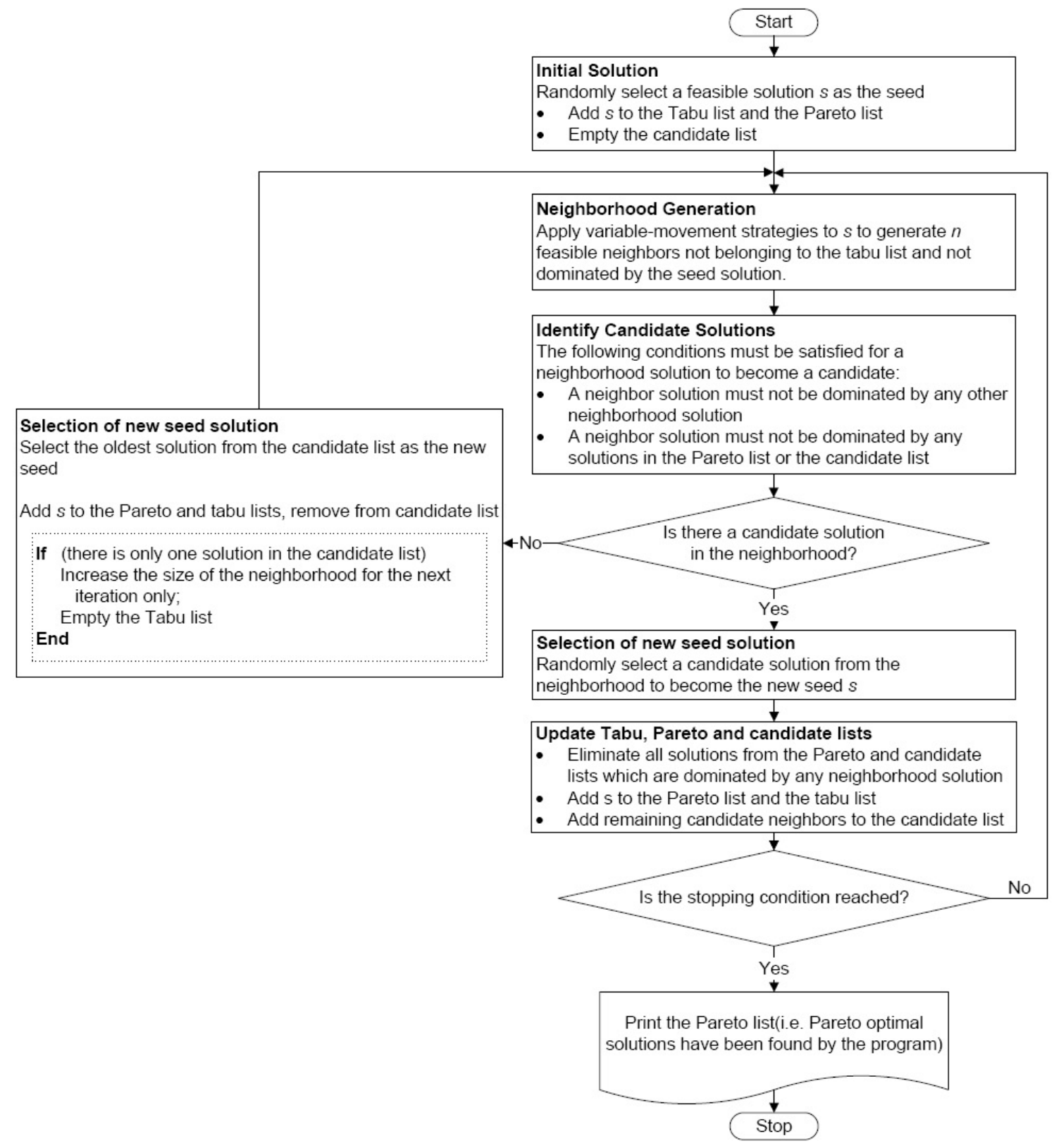

Figure 3.1: The flowchart of the modified MOTS algorithm.

It is also worth mentioning that the new independence measure adopted in this work provides a straightforward way to drive down the number of potential configurations by utilizing prior knowledge. According to the independence criterion (Section 3.2.1), particularly $S$, the sensor array should be designed in such a way that the $i^{\text {th }}$ sensor is most responsive to the $i^{\text {th }}(i=1,2, \ldots, p)$ analyte of interest. Hence, the candidates for the $i^{\text {th }}$ array com- 
ponent should not be all the $K$ sensors, but rather should be limited to a subset of the $K$ sensors that are most responsive to analyte $i$. This constraint could substantially decrease the number of candidate array configurations, and accelerate the search by the MOTS. A rough idea about the relative responsiveness of each senor to the target analytes is usually available since detailed simulation models quantifying the sensor responses to the analytes are assumed given in this work.

To conclude this section, we restate that in this thesis, the optimum design of sensor arrays is formulated as a MOO problem, to which the solutions are a set of diverse designs close to the Pareto optimal front. The design optimization problem is solved by the modified Baykasoglu's MOTS algorithm, and the evaluation of each design is based on its multivariate calibration model estimated via simulation. 


\section{Chapter 4}

\section{Empirical Results}

This section intends to demonstrate the use of the proposed methods in selecting the best configurations for the conductive polymer sensor (chemiresistors) arrays designed to detect gaseous analytes. Two simple cases were investigated: one assumes a linear calibration model (Section 4.2), and the other involves nonlinear $\mathbf{c}-\mathbf{r}$ behavior (Section 4.3). Note that these small-scale design examples, for which the potential alternatives are limited and can be easily exhausted, are intended to illustrate the proposed optimum design methods without reflecting the actual size of array design problems.

\subsection{Simulation Model}

In this work, the evaluation of sensor arrays relies on simulation rather than real experiments. In current literature, the simulation model for the conductive polymer composite sensors (chemiresistors) is well discussed. A chemiresistor responds to analytes by producing a change in the composite resistivity in exposure to a vapor environment containing the analytes, and the sensor responses depend on the conductive and thermodynamic properties of the composite and analytes. To simulate the responses of a chemiresistor, Lei et al. [2] developed a model consisting of two sub-models, as illustrated in Figure 4.1. The thermodynamic model relates the vapor concentration to the volume fraction of each component (the polymer and the analytes absorbed by the polymer) in the composite. The conductivity model associates the volume fraction of the different components in the the composite with the resistivity of the composite, which is the sensor signal. With these two sub-models 


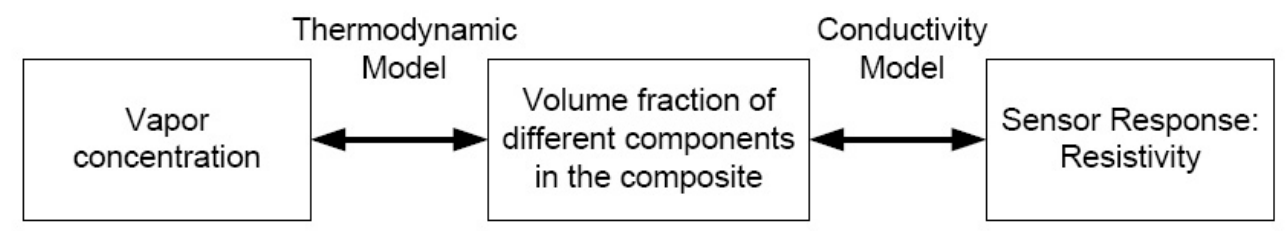

Figure 4.1: The relationship between the two sub-models for the chemiresistor.

combined, the relationship between analyte concentrations in the vapor and the resistance changes in the composite sensor can be simulated.

In Section 4.1.1, we briefly describe the simulation model in Lei et al. [2], which assumes that the sensor response to a mixture of several analytes is a linear accumulation of the influence from each individual analyte. In Section 4.1.2, we inherit the sub-model scheme (Figure 4.1), and adapt Lei et al.'s model to accommodate the interacting effects between two analytes due to their co-existence in a sample. The models in Section 4.1.1 and Section 4.1.2 will be referred to as linear and nonlinear simulation models respectively. All the programs are coded in Matlab language, as in Appendix A.

\subsubsection{The Linear Simulation Model}

The simulation model of Lei et al. [2] is based on the assumption that in the presence of more than one analyte, each analyte acts independently and the resulting sensor signal is a linear accumulation of the effects of multiple analytes. Hence, the basic building block of the simulation model in Lei et al. [2] is an analyte-polymer binary system, for which the two sub-models are detailed as follows.

\section{Thermodynamic Model}

The thermodynamic model is based on three assumptions [2]: air absorbed by the carbon black does not change the resistivity of the carbon black; non-reactive carrier gas (e.g., air) 
has negligible solubility in polymer and thus no effect on polymer swelling in the composite; and polymer swelling reaches equilibrium.

For an analyte-polymer binary system, the relationship between the vapor concentration, which is measured as the activity $a_{\text {analyte }}$, and the volume fraction of the analyte absorbed by the composite, which is denoted by $\phi_{\text {analyte }}$, is quantified by the following equation:

$$
\ln \left(a_{\text {analyte }}\right)=\ln \phi_{\text {analyte }}+\left(1-\frac{V_{\text {analyte }}}{V_{\text {polymer }}}\right)\left(1-\phi_{\text {analyte }}\right)+\chi\left(1-\phi_{\text {analyte }}\right)^{2} .
$$

$V_{\text {analyte }}$ and $V_{\text {polymer }}$ represent the molar volume of the analyte and polymer respectively, and

$\chi$ is the analyte-polymer interaction parameter. Equation (4.1) was derived by Tompa [22] based on the Flory-Huggins theory for polymer solutions. The parameters involved in (4.1) can be obtained from published literature [23, 24].

\section{Conductivity Model}

The conductivity model associates the composite resistivity $\rho_{m}$ (the sensor response) with the volume fraction of the analyte/polymer in the composite through the general effective media (GEM) equation developed by McLachlan [25]. The GEM equation is given as:

$$
\begin{array}{r}
\frac{\rho_{c}\left(\rho_{c}^{-1 / k}-\rho_{m}^{-1 / k}\right)}{\rho_{c}^{-1 / k}+C \rho_{m}^{-1 / k}}+\frac{\left(1-\rho_{c}\right)\left(\rho_{p}^{-1 / k}-\rho_{m}^{-1 / k}\right)}{\rho_{p}^{-1 / k}+C \rho_{m}^{-1 / k}}=0 \\
\text { with } C=\frac{1-\phi_{\text {critical }}}{\phi_{\text {critical }}}
\end{array}
$$

where $\phi_{\text {critical }}$ is the critical volume fraction of the carbon black or percolation threshold, $\rho_{c}$ the resistivity of the carbon black, $\rho_{p}$ the resistivity of the polymer, $\phi_{c}$ the volume fraction of the carbon black, $1-\phi_{c}$ the sum of volume fraction of polymer and absorbed analyte, and $k$ a constant related to the physical structure of the sensor (e.g. the surface area). 
In an analyte-polymer binary system, volume fraction of analyte $\phi_{\text {analytes }}$ absorbed in the composite and the volume fraction of the carbon black has the following relationship [2]:

$$
\phi_{\text {analyte }}=\frac{\phi_{0 c}-\phi_{c}}{\left(1-\phi_{c}\right) \phi_{0 c}}
$$

where $\phi_{0 c}$ is the volume fraction of the carbon black in the dry composite.

Combining (4.2) and (4.3), the relationship between the volume fraction of analyte $\phi_{\text {analytes }}$ and the composite resistivity $\rho_{m}$ is established. All the parameters involved in (4.2) and (4.3) can be obtained from the empirical results in the existing literature.

\subsubsection{The Nonlinear Simulation Model}

We adapted the linear simulation model in Lei et al. [2] to accommodate the interacting effects due to the co-existence of two analytes in a gas environment. Thus, we consider a system that incorporates two different types of analytes and one polymer sensor.

The thermodynamic model for such a ternary system is given as [26]:

$$
\begin{aligned}
& \ln \left(a_{1}\right)=\ln \phi_{1}+1-\phi_{1}-\frac{V_{1}}{V_{2}} \phi_{2}-\frac{V_{1}}{V_{3}} \phi_{3} \\
& +\left(\chi_{12} \phi_{2}+\chi_{13} \phi_{3}\right)\left(\phi_{2}+\phi_{3}\right)-\chi_{23} \frac{V_{1}}{V_{2}} \phi_{2} \phi_{3} \\
& \ln \left(a_{2}\right)=\ln \phi_{2}+1-\phi_{2}-\frac{V_{2}}{V_{1}} \phi_{1}-\frac{V_{2}}{V_{3}} \phi_{3} \\
& \quad+\left(\chi_{12} \frac{V_{2}}{V_{1}} \phi_{1}+\chi_{23} \phi_{3}\right)\left(\phi_{1}+\phi_{3}\right)-\chi_{13} \frac{V_{2}}{V_{1}} \phi_{1} \phi_{3} \\
& \sum \phi_{i}=1
\end{aligned}
$$

Subscripts 1, 2, and 3 refer to the first analyte, the second analyte, and the polymer respectively. Specifically, $a_{i}$ denotes the activity of the component $i, \phi_{i}$ the volume fraction of component $i$ in the composite, $V_{i}$ molar volume of component $i$, and $\chi_{i j}$ the interaction parameter between component $i$ and $j(i=1,2,3 ; j=1,2,3 ; i \neq j)$. Note that when the 


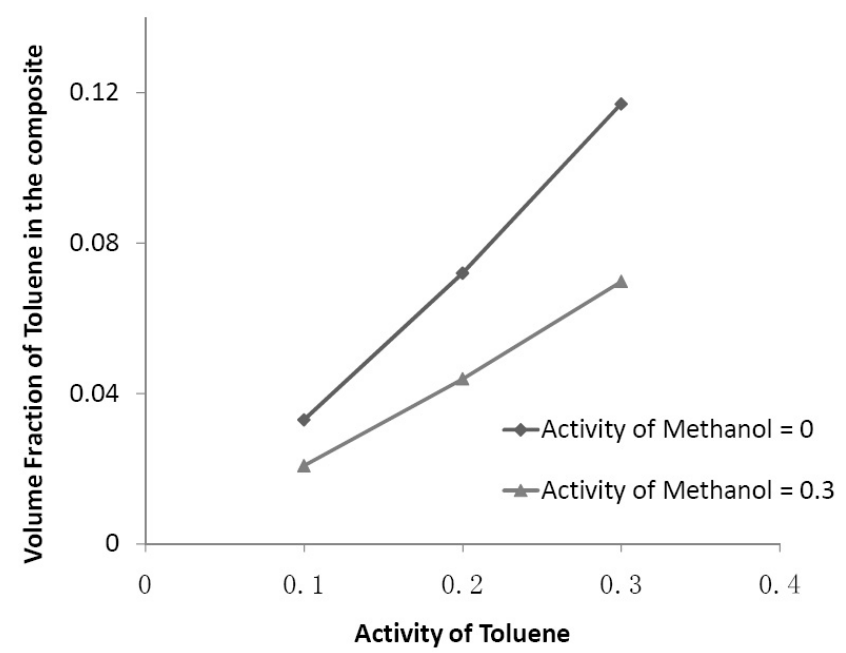

Figure 4.2: Activity of toluene with different vapor concentrations of methanol.

second analyte has little solubility in the polymer, we have $\phi_{2} \approx 0$, and the system is reduced to a binary system with equations (4.4) simplified to the form given in (4.1).

Take the toluene-methanol-PVAC ternary system as an example. Figure 4.2, which is based on the experimental data obtained in Yilmaz [26], shows that due to the presence of methanol, the solubility of toluene in PVAC is substantially lowered, and the sensitivity of toluene solubility to changes in the toluene activity is also reduced. The linear simulation model given in Section 4.1.1 is not adequate to describe the interacting effects due to the co-existence of analytes.

To the best of our knowledge, the simulation model presented here represents the first effort to simulate the sensor responses of a chemiresistor while taking into account the interacting effects of two analytes in a vapor mixture.

\subsection{Case 1: A Linear $\mathrm{c}-\mathrm{r}$ Relationship}

We consider the optimal design of a sensor array for the quantification of five target gas analytes: p-Xylene, carbon tetrachloride, acetone, n-hexane and THF. Suppose that the polymer candidates available to construct the chemiresistor array include ten materials as 
Table 4.1: Candidate polymers for the sensor array.

\begin{tabular}{|c|c|c|}
\hline Index & Polymer & Abbreviation \\
\hline 1 & poly(isobutylene) & PIB \\
\hline 2 & poly(styrene) & PS \\
\hline 3 & isotactic poly(propylene) & iPP \\
\hline 4 & poly(vinyl acetate) & PVAC \\
\hline 5 & poly(methyl methacrylate) & PMMA \\
\hline 6 & poly(ethylene glycol) & PEG \\
\hline 7 & poly(tetrahydrofuran) & PT \\
\hline 8 & linear low-density polyethylene & LLDPE \\
\hline 9 & poly(4-methyl-1-pentene) & PMP \\
\hline 10 & butadiene rubber & BR \\
\hline
\end{tabular}

given in Table 4.1. Hence, this simple design example amounts to choosing 5 among the 10 candidate polymers to build a best five-sensor array.

In this case, there are a total of $252=\left(\begin{array}{c}10 \\ 5\end{array}\right)$ possible sensor arrays. For a configuration, simulation (using the model in Section 4.1 can be performed to estimate its linear calibration model $\mathbf{r}=A \mathbf{c}$ with both $\mathbf{c}$ and $\mathbf{r}$ being $5 \times 1$ vectors. The response $\mathbf{r}$ represents the sensor signals with each element being the change of resistivity in an individual sensor. The concentration $\mathbf{c}$ is measured in terms of solvent activity, and it is restricted to the range $\left\{c_{i} \in(0,0.2) ; i=1,2, \ldots, 5\right\}$, within which a linear calibration model is adequate to capture the $\mathbf{c}-\mathbf{r}$ relationship [2]. Once the array configuration is characterized by its calibration model, it can be evaluated based on the $I$ and MRSS metrics.

For instance, for the array configured as iPP-PVAC-PMMA-PEG-PMP, simulation was performed at values of $\mathbf{c}$ determined by the two-level full factorial design [15]. From the simulation data, the calibration $\mathbf{r}=\mathbf{A c}$ is fitted as follows: 
Table 4.2: The true Pareto optimal set from an exhaustive search

\begin{tabular}{|c|c|c|c|}
\hline Index & Configurations & $I$ & $M R S S$ \\
\hline 1 & iPP-PVAC-PMMA-PEG-LLDPE & 71.686 & 10.945 \\
\hline 2 & iPP-PVAC-PMMA-PEG-PMP & 68.758 & 10.556 \\
\hline 3 & PIB-iPP-PVAC-PEG-PMP & 65.536 & 9.640 \\
\hline 4 & iPP-PVAC-PEG-LLDPE-PMP & 66.367 & 9.644 \\
\hline 5 & PIB-iPP-PVAC-PMMA-PEG & 70.676 & 10.942 \\
\hline 6 & iPP-PVAC-PMMA-PEG-BR & 135.260 & 11.224 \\
\hline
\end{tabular}

$$
\left(\begin{array}{l}
r_{1} \\
r_{2} \\
r_{3} \\
r_{4} \\
r_{5}
\end{array}\right)=\left(\begin{array}{rrrrr}
5.62 & 9.86 & 0.91 & 10.10 & 4.53 \\
11.97 & 3.11 & 8.53 & 1.02 & 7.48 \\
11.45 & 4.17 & 5.18 & 1.18 & 6.80 \\
2.62 & 7.94 & 4.36 & 0.92 & 11.48 \\
1.07 & 4.01 & 0.22 & 2.49 & 1.26
\end{array}\right)\left(\begin{array}{l}
c_{1} \\
c_{2} \\
c_{3} \\
c_{4} \\
c_{5}
\end{array}\right)
$$

The resistivity $r_{i}(i=1,2, \ldots, 5)$ is given in terms of $\Omega \cdot c m \times 10^{3}$. With the fitted model (4.5), the D matrix defined in (3.4) is simply $\mathbf{D}=\mathbf{A}$, and $I$ and MRSS can be calculated using the formulas in Section 3.2.

With the ability to evaluate an array configuration, the modified Baykasoglu's MOTS algorithm (Section 3.3.2) was used to solve (3.11) in search of the best alternatives. Since the algorithm is a probabilistic one, a different solution (configuration) set may be obtained every time it is run. To show the effectiveness of the method, we applied the MOTS algorithm on (3.11) for 100 times, and compared the solution set from each of the 100 macro-replications with the true Pareto optimal set obtained from an exhaustive search. Table 4.2 provides the six Pareto optimums and their performance metrics. Recall that the purpose of the MOTS is to obtain a solution set close to the Pareto optimal one within a reasonable amount of time. Table 4.3 summarizes the performance of the algorithm based on the 100 macroreplications. On average, by evaluating about one third of the array configurations required 
Table 4.3: The performance of the modified MOTS algorithm.

\begin{tabular}{ccc}
\hline (based on 100 macro-replications) & MOTS & Exhaustive Search \\
\hline $\begin{array}{c}\text { Average number of Pareto } \\
\text { optimums found }\end{array}$ & 4.94 & 6 \\
$\begin{array}{c}\text { Average number of array } \\
\text { configurations evaluated }\end{array}$ & 89.12 & 252 \\
\hline
\end{tabular}

by the exhaustive search, the modified MOTS is able to find five out of six Pareto optimums. In addition, out of these 100 runs only five ends up with a solution set including two or less than two Pareto optimums, while $85 \%$ of times a solution set with at least four Pareto optimums was obtained.

As pointed out in Section 3.3, the small number of non-dominant configurations resulting from the MOTS can be further judged by designers based on the relative importance of $I$ and MRSS or other performance metrics, or be put through real experiments for evaluation.

\subsection{Case 2: A Nonlinear c $-\mathrm{r}$ Relationship}

In this case, we relaxed the simplified linear assumption and accommodated the nonlinearities present in the array system. As pointed out in Section 4.1.2, such nonlinearities are due to interacting effects of co-existing analytes or nonlinear responses of sensors to pure analyte over a relatively high concentration range. We consider designing a sensor array to quantifiy two analytes, toluene and methanol. The six polymer candidates available to construct the chemiresistor array are butadiene rubber(BR), isotactic polypropylene(iPP), poly 4-methyl1-pentene(PMP), polyvinyl acetate(PVAC), polyisobutylene(PIB) and polystyrene(PS). The optimum design of a nonlinear sensor array is essentially the same as that of a linear one (Section 4.2) except that a nonlinear calibration model of the form (3.2) needs to be used for calibration and evaluation of the array. Hence, in this small example where only a total of 


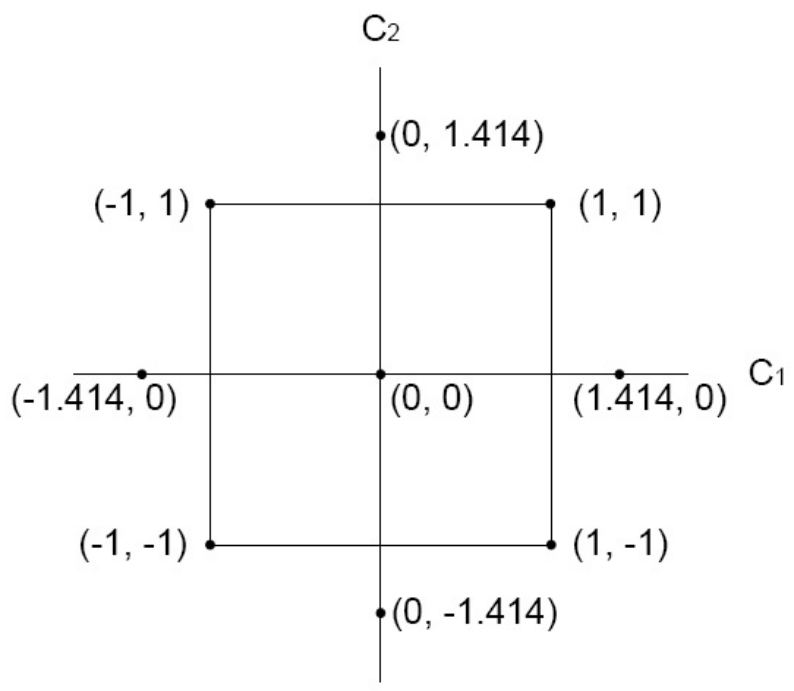

Figure 4.3: Central composite design (CCD) for nonlinear calibration model.

$15=\left(\begin{array}{l}6 \\ 2\end{array}\right)$ sensor combinations are involved, we focus on presenting the nonlinear calibration results for the purpose of illustration.

For an array configuration, the quadratic model (3.2) is used to calibrate the $\mathbf{c}-\mathbf{r}$ relationship. The analyte concentration (i.e., activity) range is set as 0 to 0.4 . To estimate the calibration model, simulation experiments are performed (using the model in Section 4.1.2) at the 9 different values of $\mathbf{c}$ following the CCD design (Section 3.1). The location of the design points in the two dimensional space of analyte concentration is shown in Figure 4.3, where the three levels of analyte activity, $0.1,0.2$, and 0.3 are coded as $-1,0$, and 1 respectively. From the simulation data collected, multivariate regression methods are used to estimate the calibration model (3.2). For instance, the fitted model for design BR-PMP is as follows (with the resistivity response given in terms of $\Omega \cdot \mathrm{cm} \times 10^{3}$ ):

$$
\begin{aligned}
& r_{1}=2.44+4.53 c_{1}+5.21 c_{2}-11.78 c_{1}^{2}-10.65 c_{2}^{2}+11.29 c_{1} c_{2} \\
& r_{2}=2.86+1.75 c_{1}-0.28 c_{2}-1.26 c_{1}^{2}+0.82 c_{2}^{2}+1.73 c_{1} c_{2}
\end{aligned}
$$


Based on the fitted calibration model (4.6) of array BR-PMP, the D matrix (3.4) is obtained as:

$$
\begin{aligned}
\mathbf{D}(\mathbf{c}) & =D_{i j}(\mathbf{c})=\left[\frac{\partial r_{i}}{\partial c_{j}}\right]_{2 \times 2} \\
& =\left(\begin{array}{ll}
4.53-23.56 c_{1}+11.29 c_{2} & 5.21+11.29 c_{1}-21.30 c_{2} \\
1.75-2.52 c_{1}+1.73 c_{2} & -0.28+1.73 c_{1}+1.64 c_{2}
\end{array}\right)
\end{aligned}
$$

Unlike the linear case (Section 4.2) where $\mathbf{D}=\mathbf{A}$, which is independent of analyte concentrations, the matrix $\mathbf{D}$ for the quadratic model here depends on the value of $\mathbf{c}$. Hence the matrix D (4.7), and thus the evaluation criteria, can only be numerically evaluated at a given analyte concentration.

To compare different configurations based on these criteria, a set of values for $\mathbf{c}$, say $\left\{\mathbf{c}_{1}, \mathbf{c}_{2}, \ldots, \mathbf{c}_{n}\right\}$, has to be selected and used to compute $\mathbf{D}(\mathbf{c})$ and subsequently the measures $I(\mathbf{c})$, and MRSS(c). The selected values of $\mathbf{c}$ should be representative in the range of analyte concentrations considered, and the evaluation criteria could be set as the (weighted) average of the measures across the set $\left\{\mathbf{c}_{1}, \mathbf{c}_{2}, \ldots, \mathbf{c}_{n}\right\}$. In this example, it is assumed that a single concentration point, say $\mathbf{c}=(0.2,0.2)$ is of particular interest, and all the system configurations are evaluated based on their performance at this analyte concentration.

Table 4.4 provides the performance metrics, evaluated at $\mathbf{c}=(0.2,0.2)$, for all the 5 Pareto optimal array configurations by the selection of our modified MOTS algorithm. Based on 100 macro-replications of our modified algorithm in the nonlinear sensor optimization problem, the average number of Pareto optimums found is 3.74, with the average evaluation times equal to 13.03. Our modified MOTS algorithm is still an acceptable method in this small case of nonlinear sensor designs. If the size of optional sensors or target analytes increases, the MOTS will work better with less evaluation times.

From the Pareto optimal set in Table 4.4, it can be seen that the designs that excel in terms of $I$, the independence criteria, have low sensitivity corresponding to low $M R S S$. 
Table 4.4: The Pareto optimal set (nonlinear case).

\begin{tabular}{|c|c|c|c|}
\hline Index & Configurations & I & MRSS \\
\hline 1 & BR-PMP & 3.20 & 2.61 \\
\hline 2 & BR-PVAC & 7.05 & 9.15 \\
\hline 3 & iPP-PMP & 6.15 & 5.38 \\
\hline 4 & PMP-PIB & 3.85 & 3.14 \\
\hline 5 & PVAC-PS & 8.91 & 11.35 \\
\hline
\end{tabular}

Table 4.5: The improvement of search results after our modification.

\begin{tabular}{ccc}
\hline (based on 100 macro-replications) & Original MOTS & Modified MOTS \\
\hline $\begin{array}{c}\text { Average number of Pareto } \\
\text { optimums found }\end{array}$ & 0.92 & 4.94 \\
$\begin{array}{c}\text { Percentage of } \\
\text { premature termination }\end{array}$ & $88 \%$ & $5 \%$ \\
\hline
\end{tabular}

Thus, trade-off decisions have to be made among the given Pareto optimums, or new sensor materials have to be included for the array construction.

\subsection{Efficiency of the modified MOTS algorithm}

In this section, the performance comparison will be made between the original and modified MOTS algorithm. All results are based on 100 macro-replications of the linear-case sensor designs in Section 4.2.

After fully implementing Baykasoglu's algorithm, we can find the search results are not deterministic, because of the stochastic factor during the selection of seed solutions from the candidate list, and the randomly neighborhood generation process. However, the search results of the original algorithm vary greatly and often give only two or less optimal solutions. This is an unacceptable drawback, as shown in Table 4.5.

In the original algorithm, the neighbor size is fixed to be $n$ each iteration. When searching in promising regions, the algorithm can keep running with new optimal solutions 


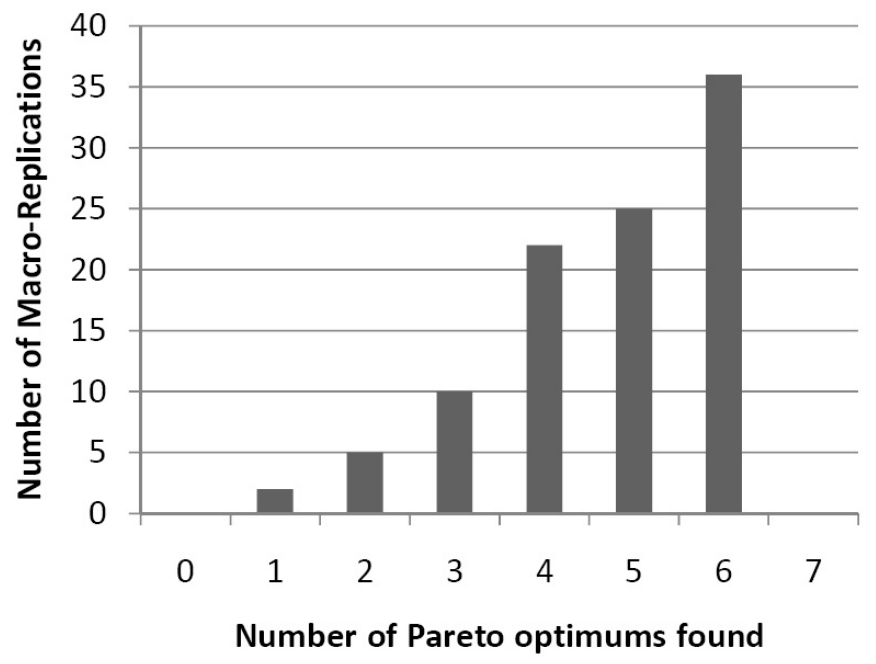

Figure 4.4: The distribution of the number of Pareto optimums found (Modified MOTS).

discovered. However, when reaching the local optima, it is very common to get no candidate solution from one iteration's neighborhood search, especially with this restrictive search scheme. In original Baykasoglu's method, the candidate list is expected to work at this moment, providing new seed solutions and helping escaping the local optima. Unfortunately, the solutions in the candidate list are often discovered from the same iteration, i.e. they are not diversified enough to reach new promising region.

In light of this, our modifications come with a simple but very effective idea. In the modified algorithm, there are two ways to escape when trapped in local optima: (i) temporarily increase the neighbor size (ii) empty the tabu list. Both of them provide a more aggressive searching scheme before a possible early termination. Since this scheme might cost additional evaluations, we can limit the times of using these modifications to control the overall evaluation times. The procedure of this modified part is described in the dotted square in Figure 3.1. In Table 4.5, there is a great improvement made by this modification.

In addition, Figure 4.4 and Figure 4.5 show the distributions of the number of Pareto optimums found, and the number of array configurations evaluated in the 100 macroreplications of our modified MOTS algorithm, respectively. Out of these 100 runs only five ends up with a solution set including two or less than two Pareto optimums, while $85 \%$ 


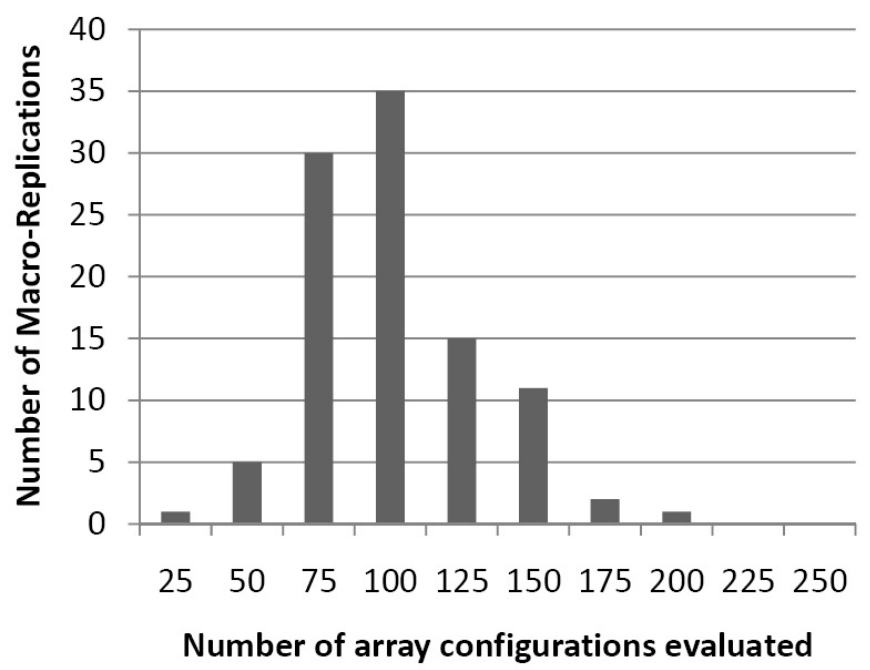

Figure 4.5: The distribution of the number of configurations evaluated (Modified MOTS). of times a solution set with at least four Pareto optimums was obtained. Also, compared to the total number of configurations, the cost of evaluation times is mainly between 75 and 150 , with a very small possibility of time-wasting in calculation. The Matlab program implementing this modified MOTS algorithm is included in Appendix C. 


\section{Chapter 5}

\section{Conclusion}

This thesis integrates a simulation model, experimental design techniques, multivariate regression, a set of new system design criteria, and a multi-objective Tabu search algorithm to approach the optimum design of sensor arrays. For illustration, the proposed methods have been applied to two small design problems, one for a linear array and the other for a nonlinear array.

Our work needs to be evaluated via a large scale of real experiments. However, various simulation results have already revealed that the multi-objective Tabu search algorithm is an valuable method in sensor optimization, when multi-criteria are needed to adequately reflect the desirable properties of a sensor array. 


\section{References}

[1] J. R. Stetter and W. Penrose. Understanding Chemical Sensors and Chemical Sensor Arrays (Electronic Noses): Past, Present, and Future, volume 10, page 189. Wiley-VCH: Weinheim, Germany, third edition, 2002.

[2] H. Lei, W. G. Pitt, L. K. McGrath, and C. K. Hob. Modeling carbon black/polymer composite sensors. Sensors and Actuators B, 125:396-407, 2007.

[3] A. Lorber. Error propagation and figures of merit for quantification by solving matrix equations. Analytical Chemistry, 58(6):1167-1172, 1986.

[4] A. N. Chaudry, T. M. Hawkins, and P. J. Travers. A method for selecting an optimum sensor array. Sensors and Actuators B, 69:236-242, 2000.

[5] H. Martens and T. Naes. Multivariate Calibration. John Wiley \& Sons, Inc., 1989.

[6] J. H. Kalivas and P. M. Lang. Interrelationships between sensitivity and selectivity measures for spectroscopic analysis. Hemometrics and Intelligent Laboratory Systems, 32(2):135-149, 1996.

[7] K. Deb. Multi-Objective Optimization using Evolutionary Algorithms. John Wiley and Sons Ltd, Chichester, West Sussex PO19 8SQ, England, 2001.

[8] D. F. Jones, S. K. Mirrazavi, and M. Tamiz. Multi-objective meta heuristics: An overview of the current state-of-the-art. European Journal of Operational Research, $137: 1-9,2002$.

[9] M. P. Hansen. Tabu search for multiobjective optimization: Mots. In MCDM '97, Cape Town, South Africa, January 6-10, 1997.

[10] S. Kulturel-Konak, A. E. Smith, and B. A. Norman. Multi-objective tabu search using a multinomial probability mass function. European Journal of Operational Research, 169:918-931, 2006.

[11] A. Baykasoglu. Goal programming using multiple objective tabu search. Journal of the Operational Research Society, 52:1359-1369, 2001. 
[12] R. Sundberg. Multivariate calibration - direct and indirect regression methodology (with discussion). Scandinavian Journal of Statistics, 26:161-207, 1999.

[13] G. A. F. Seber and C. J. Wild. Nonlinear regression. Hoboken, New Jersey: John Wiley \& Sons, Inc., 2003.

[14] A. K. Srivastava and V. P. Dravid. On the performance evaluation of hybrid and monoclass sensor arrays in selective detection of vocs: A comparative study. Sensors and Actuators B, 117:244-252, 2006.

[15] R. H. Myers and D. C. Montgomery. Response Surface Methodology: Process and Product Optimization Using Designed Experiments. Wiley-Interscience, second edition, 2002.

[16] R. A. Johnson and D. W. Wichern. Applied Multivariate Statistical Analysis. Prentice Hall, sixth edition, 2007.

[17] N. P. Suh. Axiomatic Design: Advances and Applications. Oxford University Press: New York, 2001.

[18] J. Thielman and P. Ge. Applying axiomatic design theory to the evaluation and optimization of large-scale engineering systems. Journal of Engineering Design, 17 i1:1-16, 2006.

[19] J. A. Hageman, M. Streppel, R. Wehrens, and L. M. C. Buydens. Wavelength selection with tabu search. Journal of Chemometrics, 17:427-437, 2003.

[20] J. W. Gardner, P. Boilot, and E. L. Hines. Enhanced electronic nose performance by sensor selection using a new integer based genetic algorithm approach. Sensors and Actuators B, 106:114-121, 2005.

[21] M. Marth, D. Maier, U. Stahl, M. Rapp, T. Wessa, and J. Honerkamp. Optimization of surface acoustic wave sensor arrays and application to high performance liquid chromatography. Sensors and Actuators B, 61:191-198, 1999.

[22] H. Tompa. Polymer Solutions. Butterworths Scientific Publications, London, 1956.

[23] J. Brandrup, E. H. Immergut, and E. A. Grulke. Polymer Handbook. Wiley, New York, 1999.

[24] R. P. Danner and M. S. High. Handbook of polymer solution thermodynamics. American Institute of Chemical Engineers, New York, 1993. 
[25] D. S. McLachlan, M. Blaszkiewicz, and R. E. Newnham. Electrical-resistivity of composites. J. Am. Ceram. Soc., 73:2187-2203, 1990.

[26] Y. Yurekli and S. A. Altinkaya. Measurement of ternary polymer/solvent equilibrium data by vapor-phase infrared spectroscopy. Fluid Phase Equilibria, 277:35-41, 2009. 


\section{Appendix A}

\section{The Matlab Program for the Simulation Model of Chemiresistors}

\section{A.1 The Linear Simulation Model for Chemiresistors}

At a given activity of the analyte $(a)$, the resistivity of the polymer with analytes absorbed $\left(\rho_{m}\right)$ could be calculated by the following steps:

1. Calculate the volume fraction $\phi$ of the pure analyte at the activity $a$ in the pure polymer.

2. Calculate the resistivity $\rho_{m}$ (in $\left.10^{3}\right)$ of the mixture.

Listing A.1: The Matlab program for the linear simulation model of chemiresistors

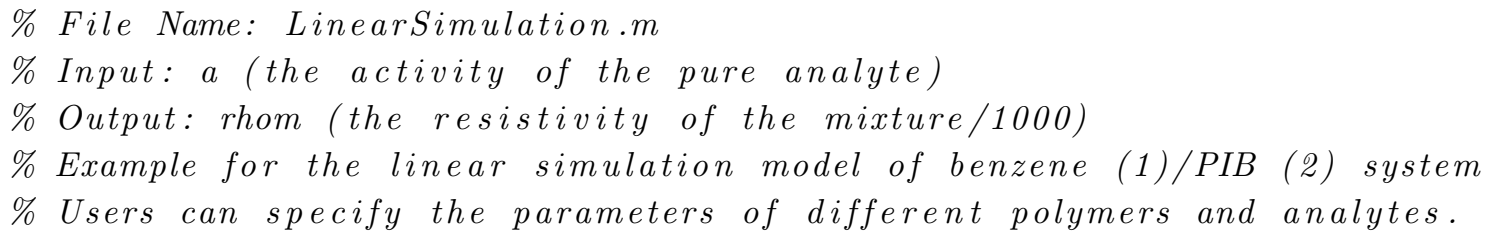




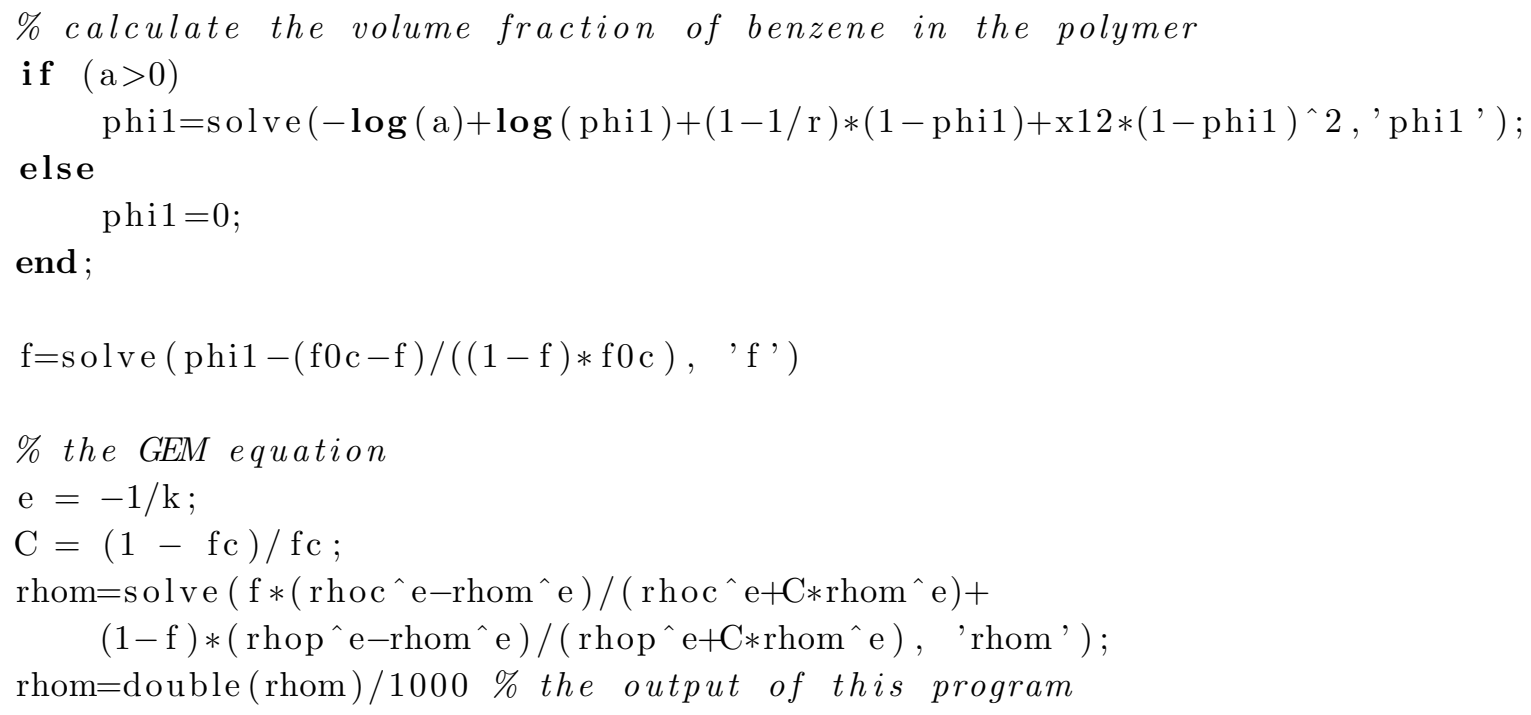




\section{A.2 The Nonlinear Simulation Model for Chemiresistors}

At given activities of the analyte $\left(a_{i}\right)$, the resistivity of the polymer with analytes absorbed $\left(\rho_{m}\right)$ could be calculated by the following steps:

1. Calculate the volume fractions $\phi_{i}$ of each pure analyte at the activity $a_{i}$ in the pure polymer from the Flory-Huggins equation.

2. Calculate the resistivity $\rho_{m}$ (in $10^{3}$ ) of the mixture.

Listing A.2: The Flory-Huggins equation to be solved in the nonlinear simulation model

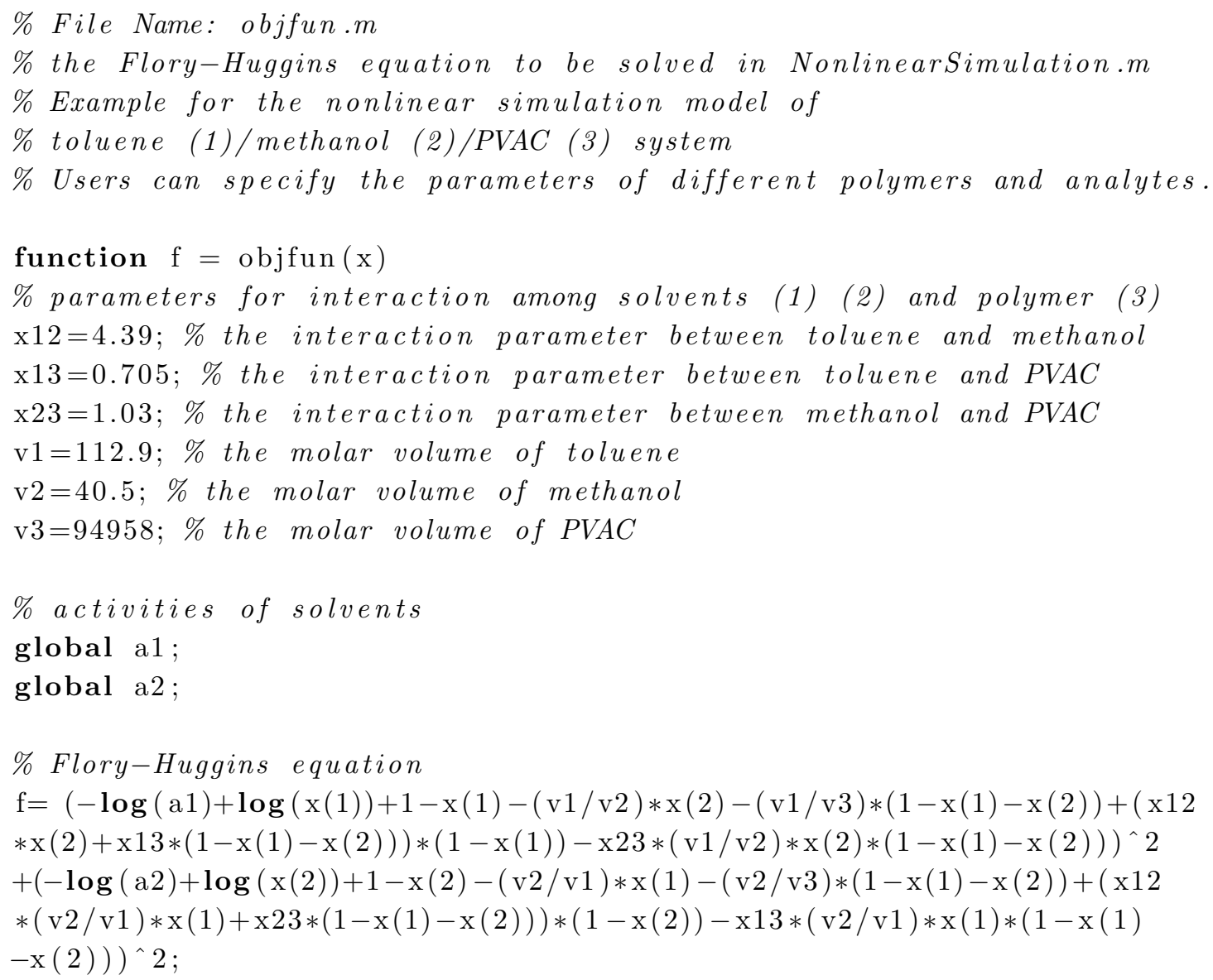


Listing A.3: The Matlab program for the nonlinear simulation model of chemiresistors

\% File Name: NonlinearSimulation.m

\% Input: $x 1$ (the activity of the pure analyte (1))

\% Input: x2 (the activity of the pure analyte (2))

$\%$ Output: rhom (the resistivity of the mixture/1000)

function NonlinearSimulation $(x 1, x 2)$

\% activities of solvents

global a1;

$\mathrm{a} 1=\mathrm{x} 1$;

global a 2 ;

$\mathrm{a} 2=\mathrm{x} 2$;

syms f rhom

$\%$ constant values, determined by the physical structure of the sensor

$\mathrm{f} 0 \mathrm{c}=0.1$;

rhop $=10^{\wedge} 16$;

$\mathrm{fc}=0.094$;

rhoc $=0.87$;

$\mathrm{k}=2.5$;

$\%$ calculate the volume fractions of toluene and methanol in the polymer $\mathrm{x} 0=[0.0001,0.0001] ; \quad \%$ start value

options=optimset ('NonlEqnAlgorithm', 'gn'); \%gauss-newton

phi $=$ fsolve(@objfun, x0, options) \%solve the Flory-Huggins equation

$\mathrm{f}=\operatorname{solve}(\operatorname{phi}(1)+\operatorname{phi}(2)-(\mathrm{f} 0 \mathrm{c}-\mathrm{f}) /((1-\mathrm{f}) * \mathrm{f} 0 \mathrm{c}), \quad$ f' $)$;

$\%$ the GEM equation

$\mathrm{e}=-1 / \mathrm{k}$;

$\mathrm{C}=(1-\mathrm{fc}) / \mathrm{fc}$;

$\operatorname{rhom}=\operatorname{solve}\left(\mathrm{f} *\left(\operatorname{rhoc}^{\wedge} \mathrm{e}-\mathrm{rhom}^{\wedge} \mathrm{e}\right) /\left(\operatorname{rhoc} \wedge \mathrm{e}+\mathrm{C} * \mathrm{rhom}^{\wedge} \mathrm{e}\right)+\right.$

$(1-\mathrm{f}) *(\operatorname{rhop} \wedge \mathrm{e}-\mathrm{rhom} \wedge \mathrm{e}) /(\operatorname{rhop} \wedge \mathrm{e}+\mathrm{C} *$ rhom^e $)$, 'rhom' $)$;

rhom=double $($ rhom $) / 1000 \%$ the output of this program 


\section{Appendix B}

\section{The Database of 10 polymers and 9 analytes}

The data in Table B.1 are the resistivity $\left(\Omega \cdot \mathrm{cm} \times 10^{3}\right)$ of each polymer at unit activity of each analyte. 


\begin{tabular}{|c|c|c|c|c|c|c|c|c|c|c|}
\hline の壱 & $\begin{array}{l}8 \\
6 \\
0 \\
11 \\
\infty\end{array}$ & 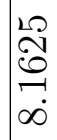 & $\mid \begin{array}{c}\stackrel{0}{N} \\
\text { în } \\
i ? \\
+i\end{array}$ & $\begin{array}{l}2 \Omega \\
10 \\
\stackrel{2}{7} \\
\end{array}$ & $\begin{array}{l}20 \\
\\
\infty \\
0 \\
0\end{array}$ & 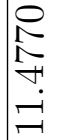 & $\left|\begin{array}{c}0 \\
0 \\
0 \\
0 \\
\sigma\end{array}\right|$ & 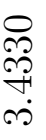 & $\begin{array}{l}\stackrel{8}{8} \\
\stackrel{0}{\circ} \\
-1 \\
-1\end{array}$ & $\begin{array}{l}0 \\
\stackrel{1}{8} \\
8 \\
\infty \\
\infty\end{array}$ \\
\hline 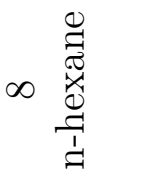 & $\begin{array}{l}0 \\
1 \\
\infty \\
0 \\
0 \\
0\end{array}$ & 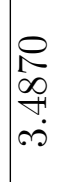 & $\mid \begin{array}{l}0 \\
0 \\
8 \\
0 \\
0 \\
0 \\
1\end{array}$ & $\begin{array}{l}R \\
\stackrel{1}{0} \\
0 \\
-i\end{array}$ & 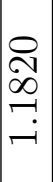 & 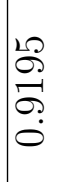 & 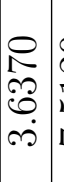 & 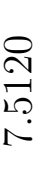 & \begin{tabular}{l}
20 \\
0 \\
$\infty$ \\
+1 \\
\multirow{1}{*}{}
\end{tabular} & 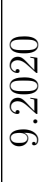 \\
\hline 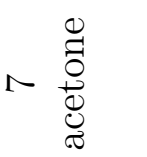 & $\begin{array}{l}8 \\
8 \\
0 \\
0 \\
0\end{array}$ & $\begin{array}{l}8 \\
0 \\
0 \\
1 \\
ن \\
\text { s. }\end{array}$ & $\mid \begin{array}{l}2 \\
0 \\
0 \\
0 \\
0 \\
0\end{array}$ & $\left|\begin{array}{c}1 \\
\stackrel{1}{2} \\
N \\
i \\
\infty \\
\infty\end{array}\right|$ & $\begin{array}{l}20 \\
\infty \\
\infty \\
-1 \\
20\end{array}$ & $\begin{array}{l}10 \\
6 \\
10 \\
2 \\
+ \\
+1\end{array}$ & 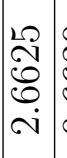 & $\begin{array}{l}0 \\
0 \\
0 \\
0 \\
0\end{array}$ & $\begin{array}{l}\stackrel{2}{\Omega} \\
\stackrel{\leftrightarrow}{\sim} \\
\stackrel{0}{0}\end{array}$ & 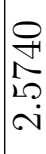 \\
\hline ○ & 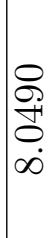 & $\begin{array}{l}10 \\
60 \\
1 \\
0 \\
0\end{array}$ & $\mid$ & 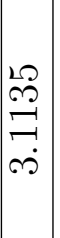 & $\begin{array}{l}8 \\
8 \\
0 \\
-1 \\
+4\end{array}$ & $\begin{array}{l}8 \\
6 \\
-0 \\
0 \\
1\end{array}$ & $\mid \begin{array}{l}12 \\
20 \\
0 \\
1 \\
0 \\
0 \\
0\end{array}$ & $\begin{array}{l}8 \\
\stackrel{0}{0} \\
\stackrel{1}{0} \\
\infty\end{array}$ & 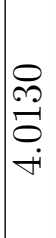 & 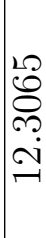 \\
\hline 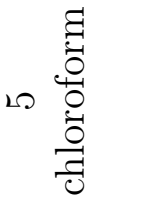 & $\begin{array}{l}R \\
0 \\
0 \\
0 \\
10\end{array}$ & 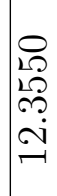 & $\mid \begin{array}{l}20 \\
2 \\
\infty \\
0 \\
0 \\
0\end{array}$ & $\left|\begin{array}{c}\infty \\
\infty \\
\infty \\
N \\
N \\
N \\
N\end{array}\right|$ & 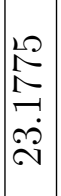 & 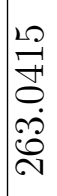 & 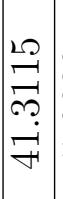 & $\begin{array}{l}8 \\
\infty \\
0 \\
0 \\
i \dot{0}\end{array}$ & 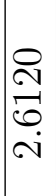 & $\begin{array}{l}\stackrel{2}{2} \\
\stackrel{2}{\rightleftharpoons} \\
\stackrel{\infty}{\infty}\end{array}$ \\
\hline 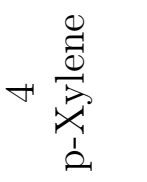 & $\begin{array}{l}8 \\
\infty \\
\sim \\
i \\
\infty \\
\infty\end{array}$ & \begin{tabular}{l}
$\stackrel{\rho}{\infty}$ \\
$\infty$ \\
-1 \\
0 \\
\hdashline
\end{tabular} & $\left|\begin{array}{c}\mathscr{8} \\
\mathbb{8} \\
0 \\
\dot{1} \\
\dot{0}\end{array}\right|$ & 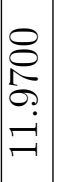 & 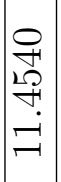 & 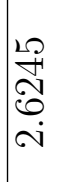 & $\frac{8}{0}$ & $\begin{array}{l}10 \\
\infty \\
\curvearrowright \\
0 \\
\infty \\
0\end{array}$ & 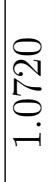 & 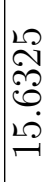 \\
\hline 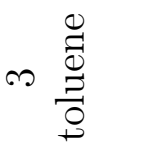 & $\begin{array}{l}\stackrel{10}{+} \\
+\infty \\
\infty \\
\infty\end{array}$ & \begin{tabular}{l}
$\qquad 0$ \\
\multirow{2}{*}{} \\
0 \\
0 \\
0 \\
1
\end{tabular} & $\mid \begin{array}{l}20 \\
70 \\
0 \\
20 \\
20\end{array}$ & $\left|\begin{array}{c}10 \\
0 \\
\infty \\
0 \\
+1 \\
0 \\
-1\end{array}\right|$ & 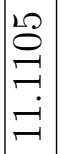 & $\begin{array}{l}20 \\
0 \\
6 \\
1 \\
7\end{array}$ & 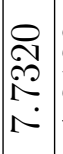 & $\begin{array}{l}\stackrel{\ominus}{\sim} \\
\stackrel{\sim}{\sim} \\
\stackrel{+}{+}\end{array}$ & 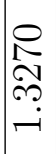 & 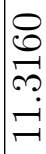 \\
\hline 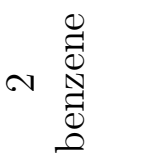 & $\begin{array}{l}10 \\
\infty \\
20 \\
\infty \\
\infty \\
\infty\end{array}$ & $\begin{array}{l}20 \\
2 \\
N \\
\\
0\end{array}$ & $\mid \begin{array}{l}10 \\
0 \\
0 \\
7 \\
10 \\
10\end{array}$ & $\mid$\begin{tabular}{l}
0 \\
1 \\
0 \\
0 \\
0 \\
\hdashline \\
-1
\end{tabular} & 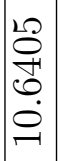 & $\begin{array}{l}0 \\
0 \\
\infty \\
\infty \\
\infty\end{array}$ & $\begin{array}{l}0 \\
\tilde{N} \\
\tilde{\sigma} \\
\sigma \\
\sigma\end{array}$ & 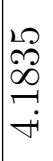 & $\begin{array}{l}20 \\
\infty \\
0 \\
2 \\
20 \\
-1 \\
-1\end{array}$ & $\begin{array}{l}\stackrel{\rho}{\sim} \\
\stackrel{2}{\infty} \\
\infty\end{array}$ \\
\hline$-\frac{\ddot{\Phi}}{\stackrel{\pi}{*}}$ & $\begin{array}{l}8 \\
8 \\
8 \\
0\end{array}$ & $\begin{array}{l}8 \\
8 \\
8 \\
0 \\
0\end{array}$ & $\mid \begin{array}{l}8 \\
8 \\
8 \\
0 \\
0\end{array}$ & $\mid \begin{array}{l}10 \\
\stackrel{F}{7} \\
3 \\
0\end{array}$ & $\mid \begin{array}{l}8 \\
0 \\
0 \\
0 \\
0 \\
0\end{array}$ & $\begin{array}{c}尺 \\
1 \\
0 \\
0 \\
0\end{array}$ & 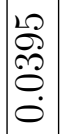 & $\begin{array}{l}8 \\
8 \\
8 \\
0 \\
0\end{array}$ & 送 & $\begin{array}{l}\mathscr{8} \\
8 \\
0 \\
0\end{array}$ \\
\hline & $\frac{\varphi}{\alpha_{1}}$ & $\tilde{\Sigma}$ & $\vec{A}$ & $\begin{array}{l}0 \\
\vdots \\
\vdots \\
2 \\
\end{array}$ & $\sum_{\infty}$ & 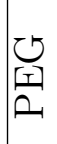 & En & 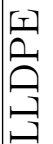 & $\sum_{1}^{\infty}$ & $\stackrel{n}{n}$ \\
\hline & $\neg$ & $\sim$ & $\infty$ & 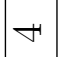 & 10 & 0 & $N$ & $\infty$ & os & $\stackrel{0}{\circ}$ \\
\hline
\end{tabular}




\section{Appendix C}

\section{The Matlab Program Applying the Modified MOTS Algorithm}

The Criteria implemented in Listing C.1 are $I$, and $M R S S$. Both criteria have been fully discussed in Section 3.2, and will be used to evaluate possible array configurations by the Matlab program in Listing C.2.

Listing C.1: Criteria used in array optimization

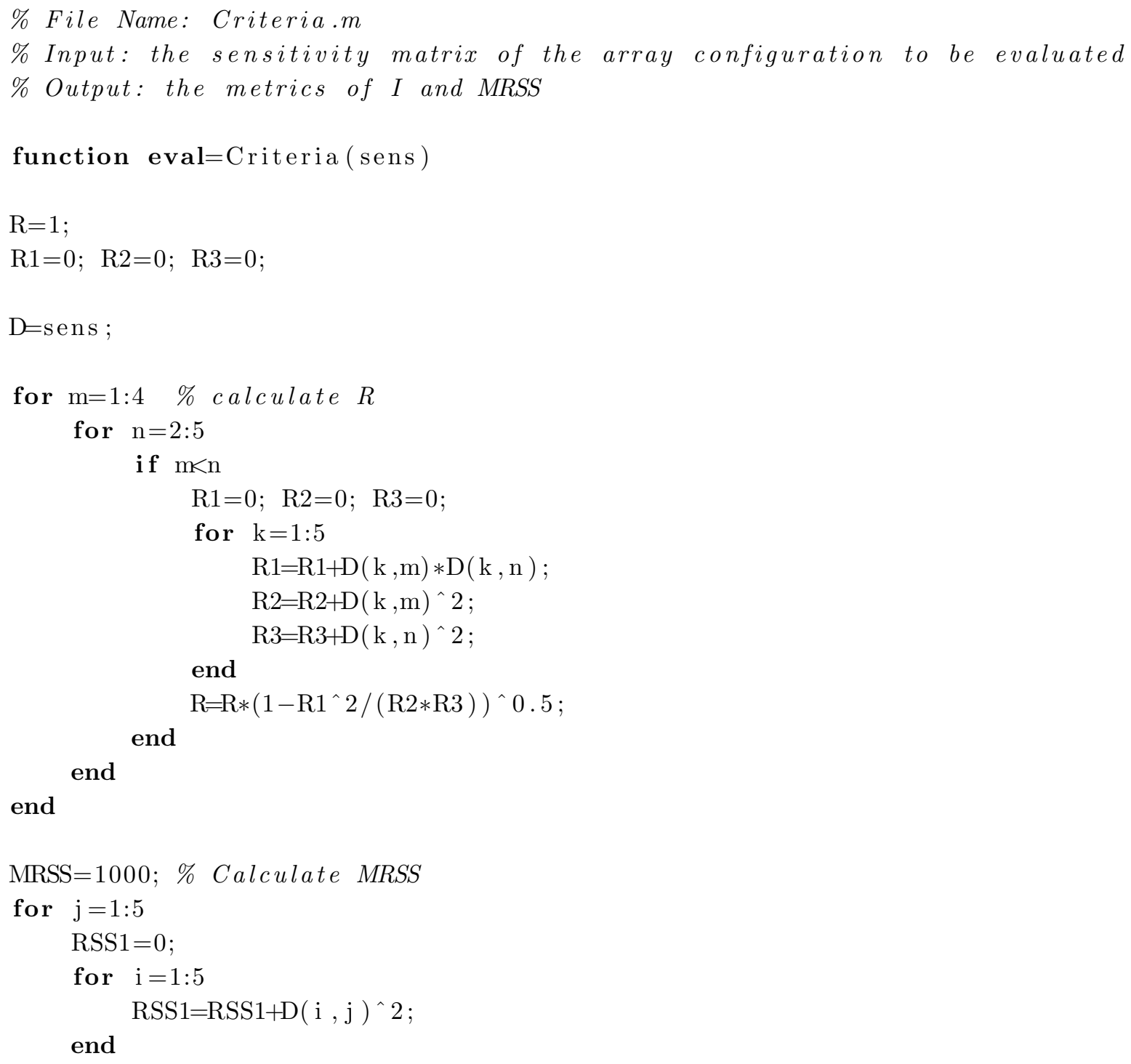




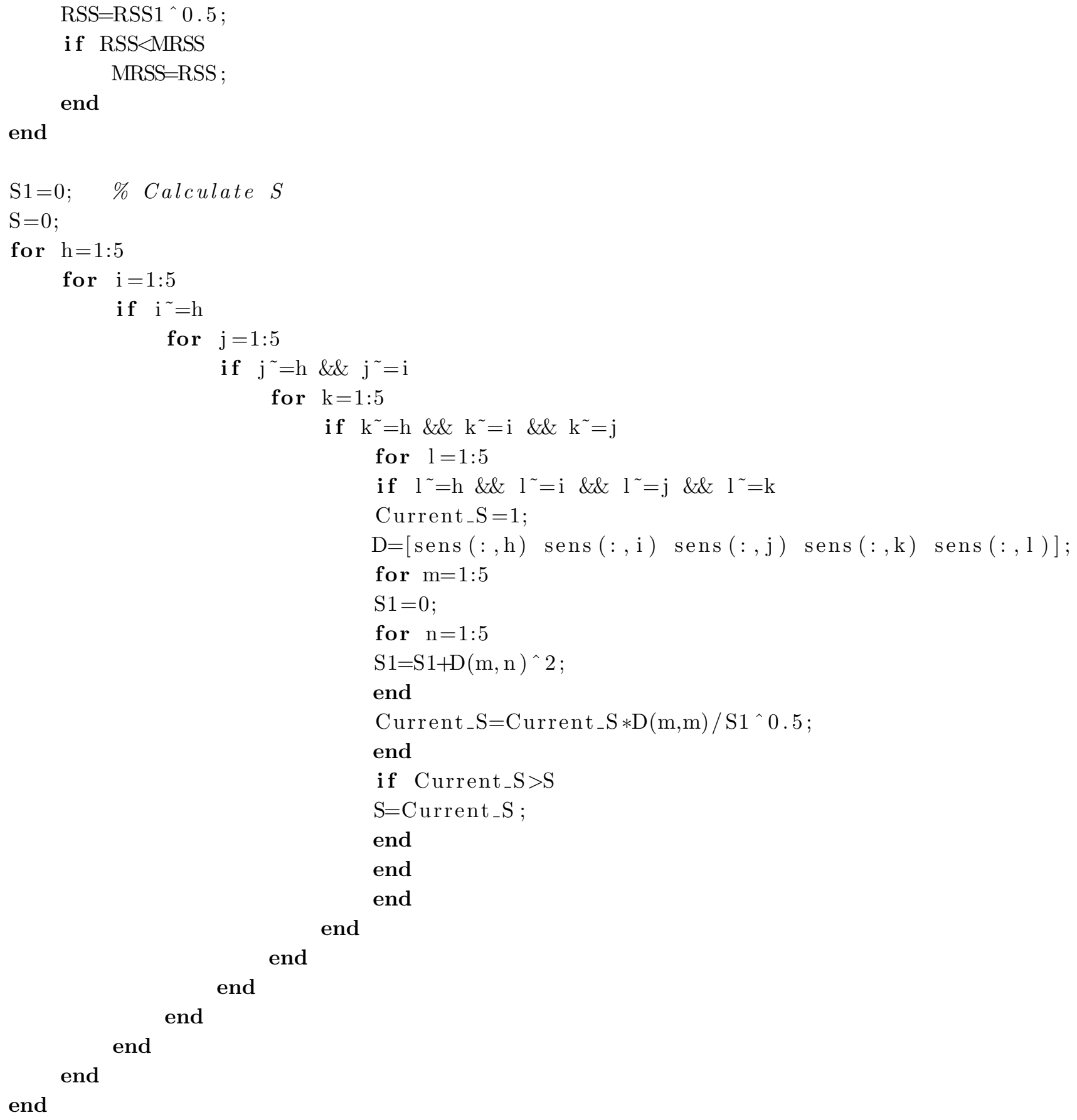


The flowchart of the Matlab program in Listing C.2 is shown in Figure 3.1. The database we use (Appendix B) includes 10 polymers and 9 analytes. To test our modified MOTS algorithm, any 5 analytes could be chosen as the target set.

Listing C.2: The Matlab program applying the modified MOTS algorithm

\% File Name: MOTS.m

\% Implement the modified MOTS algorithm

$\%$ Output: the Pareto optimums found

\section{function $\operatorname{MOTS}()$}

raw $=$ zeros $(10,9)$; \% the database of the 10 polymers and 9 analytes $\mathrm{raw}=\left[\begin{array}{llllllllll}0.0000 & 3.8585 & 3.8445 & 3.5280 & 5.3670 & 8.0490 & 0.6000 & 6.8710 & 3.1660\end{array}\right.$

$\begin{array}{lllllllll}0.0000 & 9.7795 & 10.0545 & 10.1830 & 12.3550 & 6.7665 & 3.4600 & 3.4870 & 8.1625\end{array}$

$\begin{array}{lllllllll}0.0000 & 5.4615 & 5.7645 & 5.6200 & 6.6805 & 9.8635 & 0.9085 & 10.0970 & 4.5320\end{array}$

$\begin{array}{lllllllll}0.3415 & 10.0970 & 10.4865 & 11.9700 & 27.2880 & 3.1135 & 8.5275 & 1.0170 & 7.4755\end{array}$

$\begin{array}{lllllllll}0.0790 & 10.6405 & 11.1105 & 11.4540 & 23.1775 & 4.1690 & 5.1805 & 1.1820 & 6.8015\end{array}$

$\begin{array}{lllllllll}6.1070 & 8.8610 & 4.7695 & 2.6245 & 263.0415 & 7.9360 & 4.3565 & 0.9195 & 11.4770\end{array}$

$\begin{array}{lllllllll}0.0395 & 9.9270 & 7.7320 & 5.7160 & 41.3115 & 10.7955 & 2.6625 & 3.6370 & 9.1010\end{array}$

$\begin{array}{lllllllll}0.0000 & 4.1835 & 4.2120 & 3.9285 & 5.6680 & 8.4690 & 0.6630 & 7.5120 & 3.4330\end{array}$

$\begin{array}{lllllllll}0.0000 & 1.5085 & 1.3270 & 1.0720 & 2.6120 & 4.0130 & 0.2195 & 2.4865 & 1.2600\end{array}$

$\left.\begin{array}{lllllllll}0.0000 & 8.3920 & 11.3160 & 15.6325 & 8.1435 & 12.3065 & 2.5740 & 9.2020 & 8.9010\end{array}\right] ;$

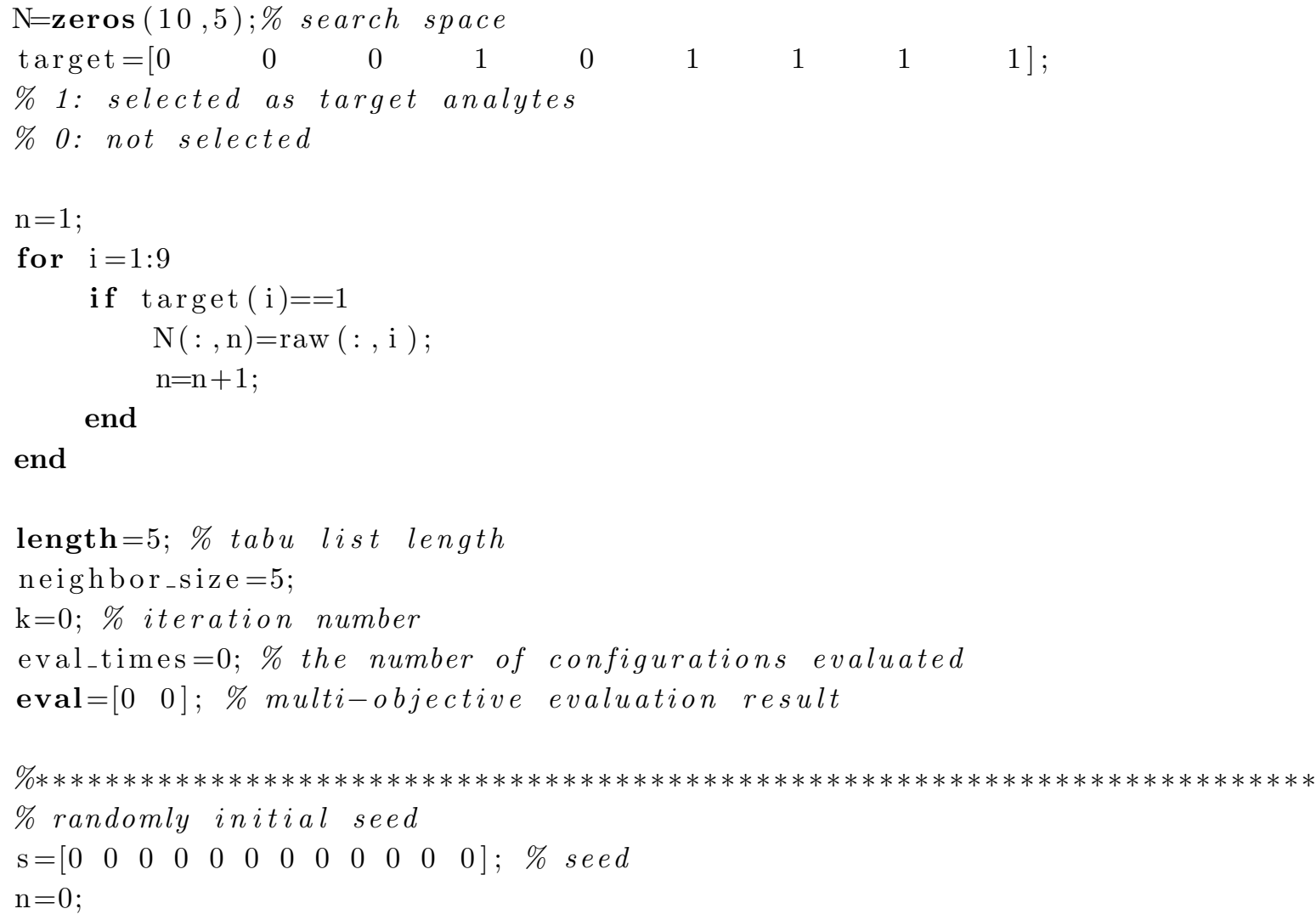


$\mathrm{i}=1$;

while $\mathrm{i}<=10$

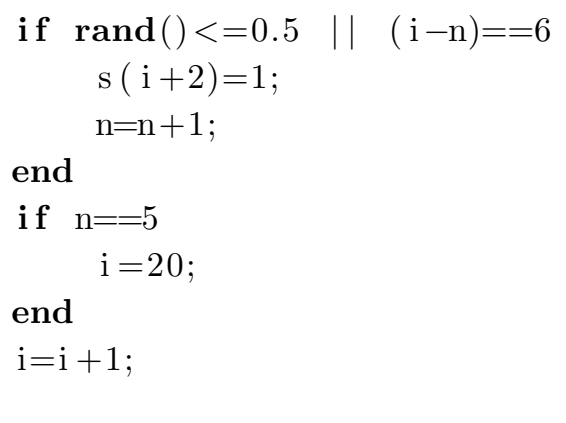

$\%$ initial tabu list, length

$\mathrm{T}=\left[\begin{array}{llllllllll}0 & 0 & 0 & 0 & 0 & 0 & 0 & 0 & 0 & 0\end{array}\right] ; \%$ Tabu list

for $i=1: 10$

$$
\text { if } \quad \begin{aligned}
\mathrm{s}(\mathrm{i}+2)==1 \\
\mathrm{~T}(\mathrm{i})=\text { length } ;
\end{aligned}
$$

end

end

$\mathrm{n}=1$;

$\operatorname{sens}=\operatorname{zeros}(5,5)$;

for $m=3: 12$

if $\mathrm{s}(\mathrm{m})==1$

$\operatorname{sens}(\mathrm{n},:)=\mathrm{N}(\mathrm{m}-2,:)$;

$\mathrm{n}=\mathrm{n}+1$;

end

end

eval $=$ Criteria ( sens) ; simulation

$\mathrm{s}(1)=\mathbf{e v a l}(1)$;

$\mathrm{s}(2)=\mathbf{e v a l}(2)$;

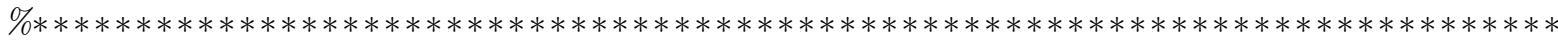

$\%$ the main search procedure

current $=\left[\begin{array}{llllllllllll}0 & 0 & 0 & 0 & 0 & 0 & 0 & 0 & 0 & 0 & 0 & 0\end{array}\right]$;

\% last 10 numbers: current search point evaluated

\% first 2 numbers: current evaluation values

Neighbor_Results=zeros $(30,12)$;

\% neighborhood size should not be greater than 30

Neighbor_Index $=0 ; \%$ Number of nondominated neighbors

Pareto_List $=$ zeros $(30,12) ; \%$ the Pareto optimum found

Pareto_Index $=0$;

Pareto_List $(1,:)=\mathrm{s}$; 


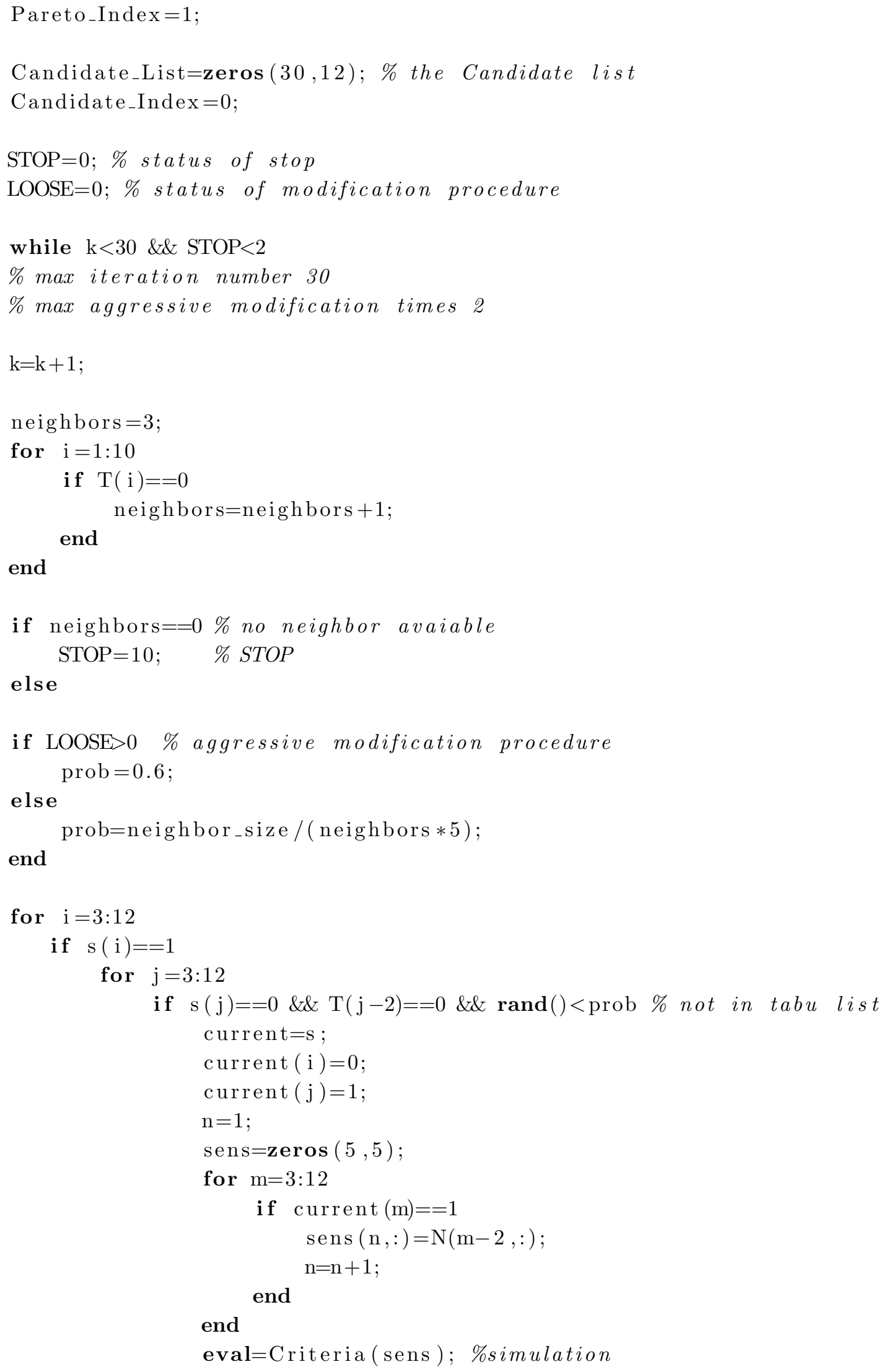




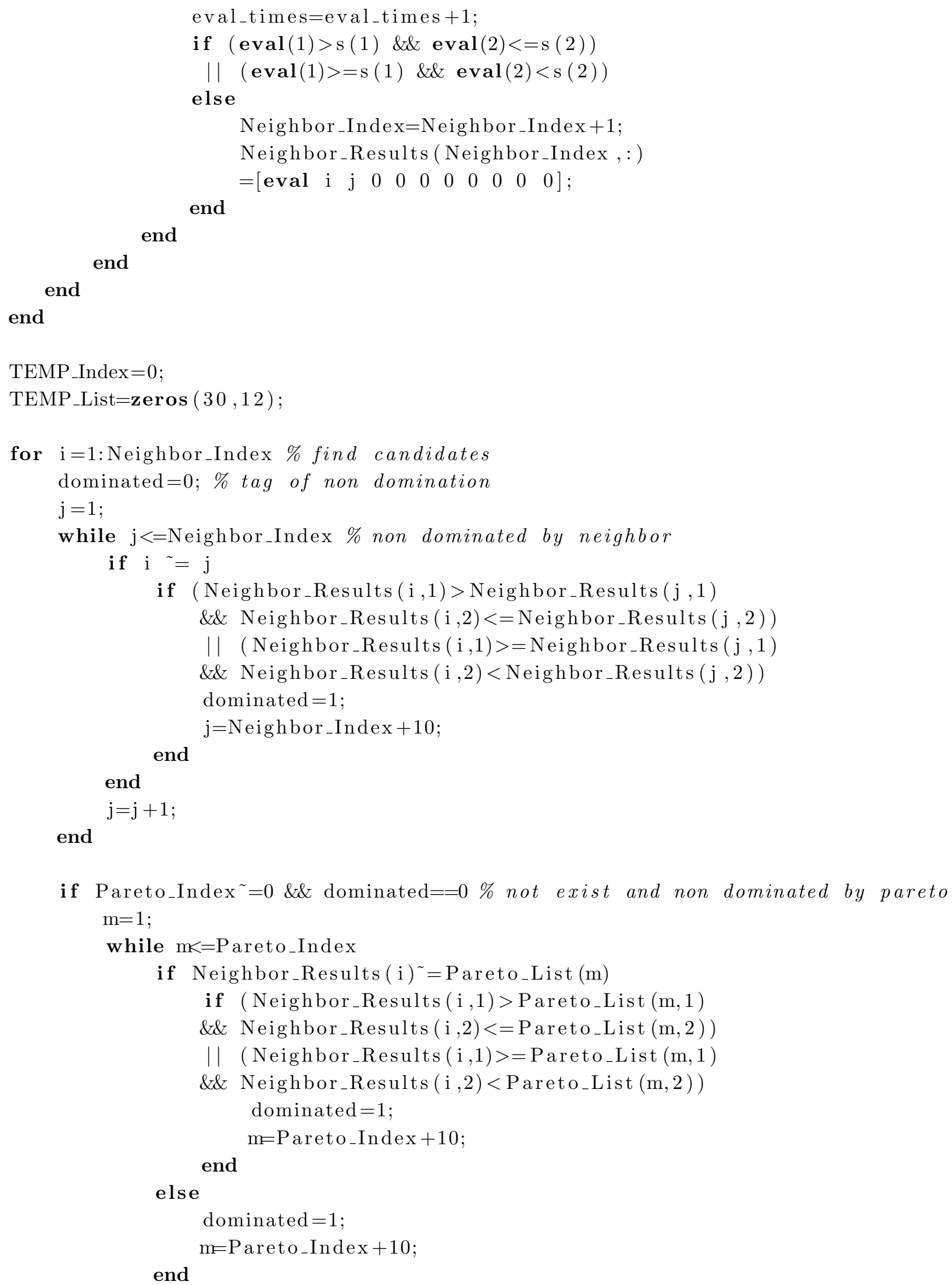




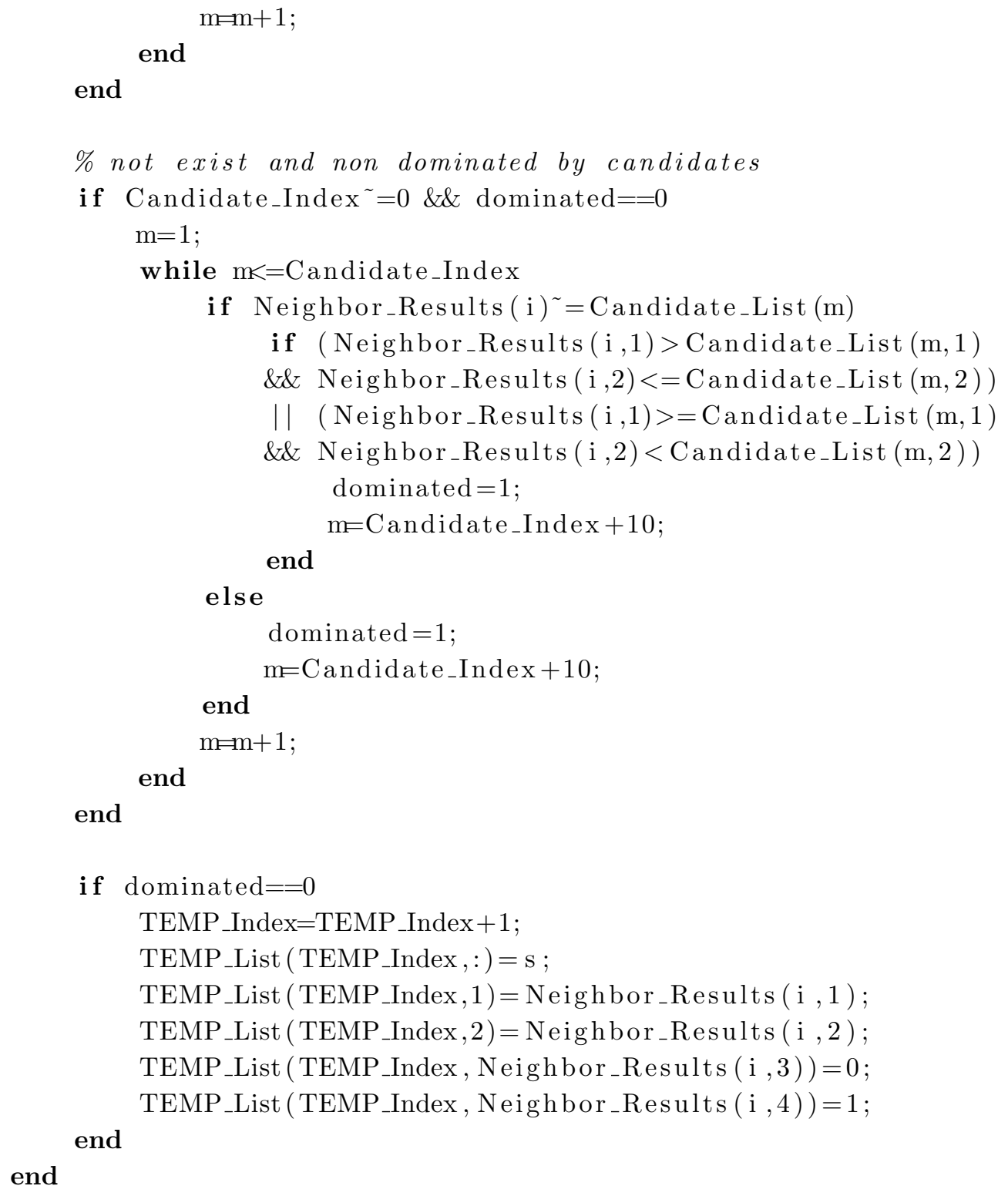

Neighbor_Results=TEMP_List; \% selected neighborhood Neighbor_Index=TEMP_Index;

if Neighbor_Index $==0 \%$ If no new candidates if Candidate_Index ${ }^{\sim}=0$

if Candidate_Index $==1$ 


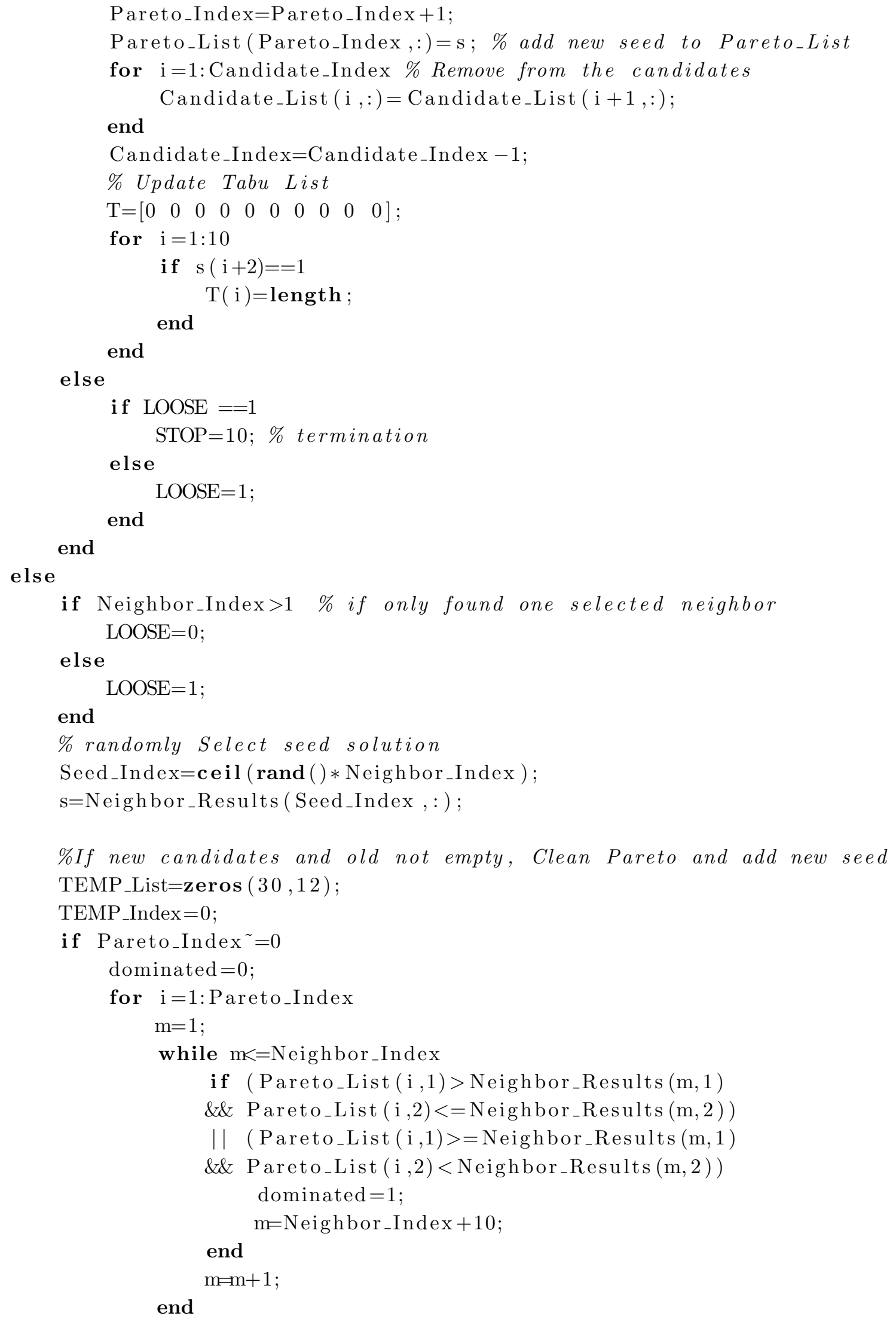




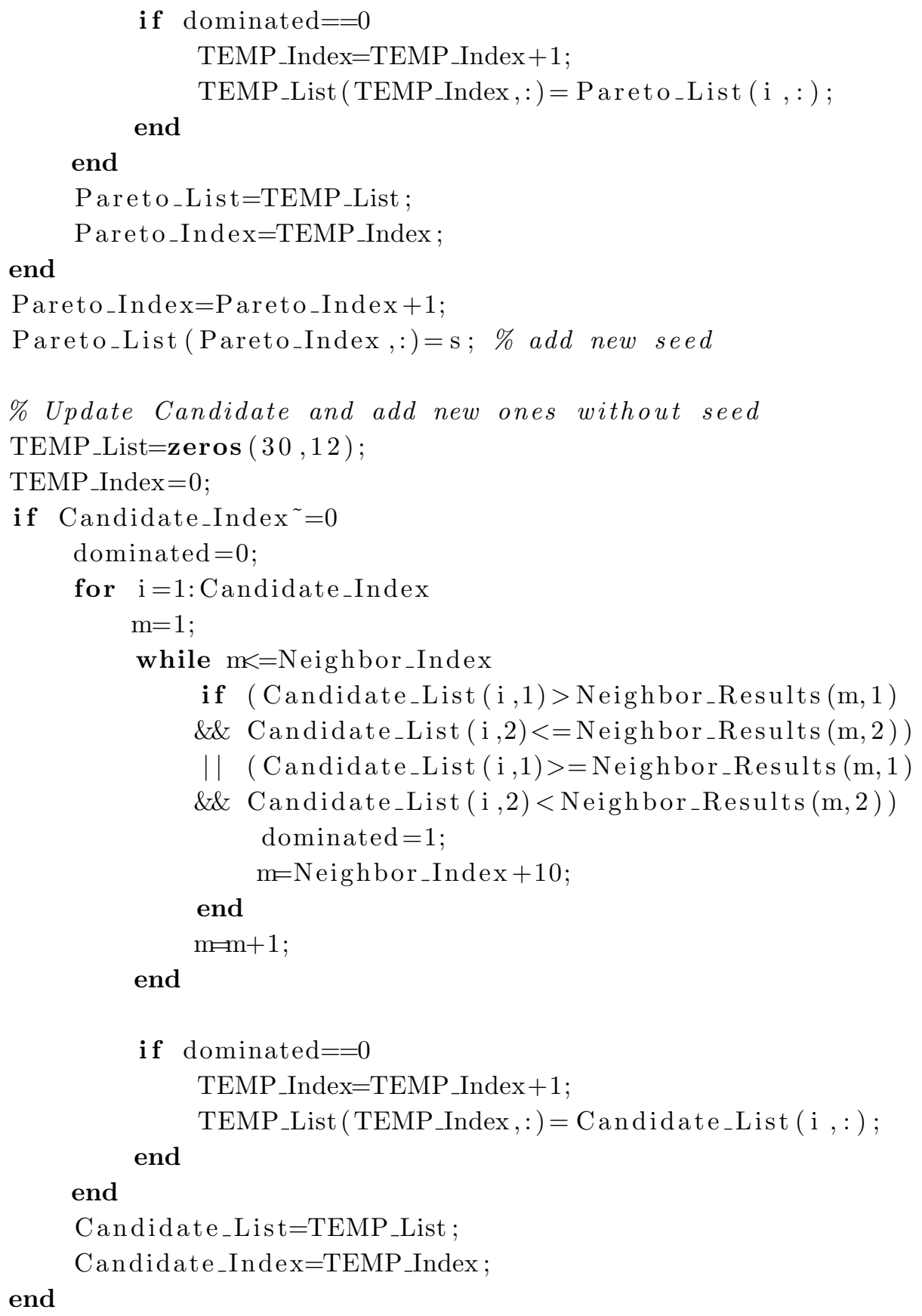




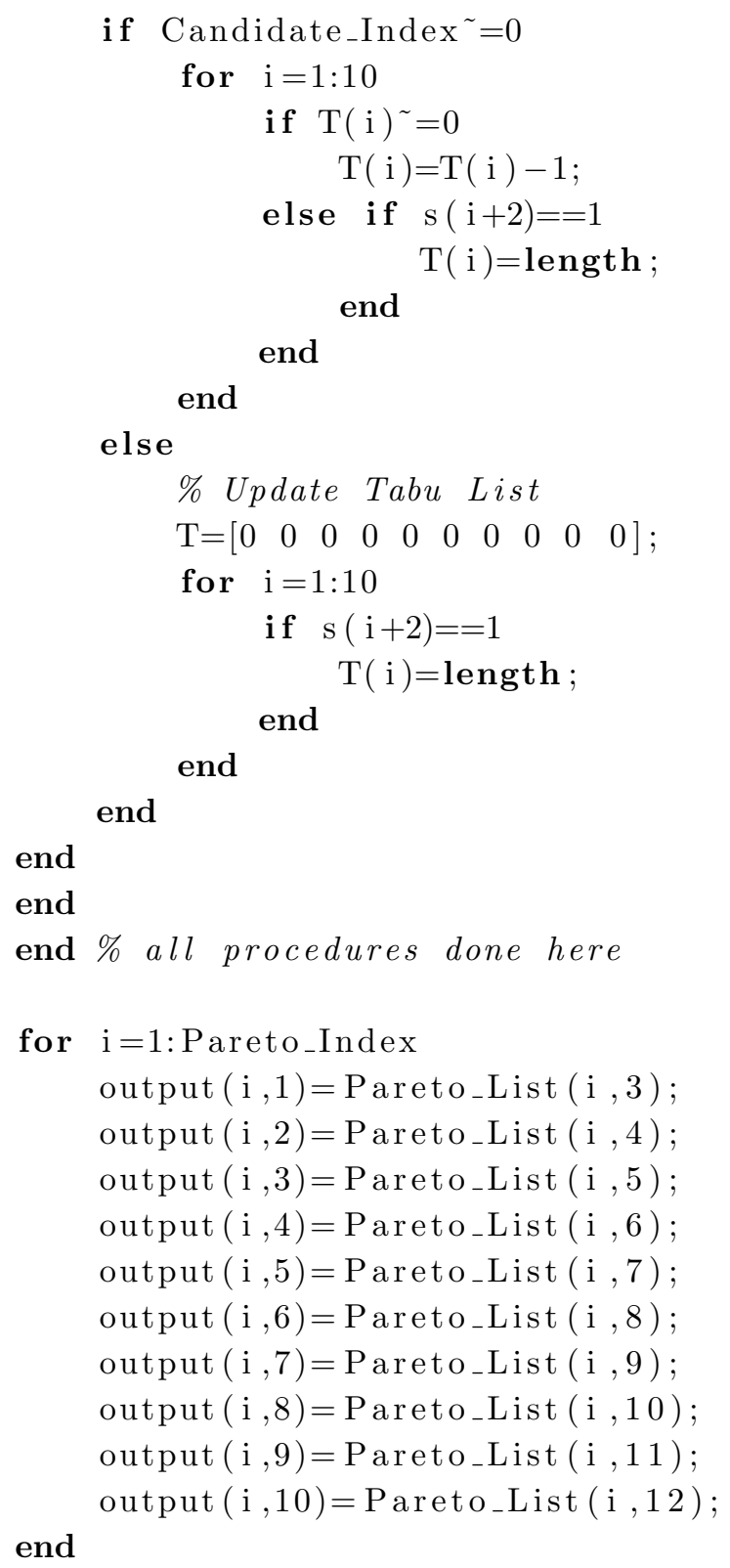

